\title{
Review
}

\section{Characteristic adaptations of the extracellular matrix in dilated cardiomyopathy}

Laura Louzao-Martinez $\mathrm{MSc}^{1,2}$, Aryan Vink MD PhD ${ }^{3}$, Magdalena Harakalova, MD PhD ${ }^{2,3,4}$, Folkert W. Asselbergs MD PhD ${ }^{2,4,5}$, Marianne C. Verhaar ${ }^{1}$ MD PhD, Caroline Cheng PhD ${ }^{1,6}$

${ }^{1}$ Department of Nephrology and Hypertension, Division Internal Medicine and Dermatology, University Medical Center Utrecht, the Netherlands; ${ }^{2}$ Netherlands Heart Institute; ${ }^{3}$ Department of Pathology, University Medical Center Utrecht, the Netherlands; ${ }^{4}$ Department of Cardiology, Division Heart and Lungs, University Medical Center Utrecht, the Netherlands; ${ }^{5}$ Institute of Cardiovascular Science, Faculty of Population Health Sciences, University College London, United Kingdom; ${ }^{6}$ Department of Cardiology, Thoraxcenter, Division of Experimental Cardiology, Erasmus University Medical Center Rotterdam, the Netherlands

Keywords: dilated cardiomyopathy (DCM); extracellular matrix (ECM); myocardial fibrosis; animal models; transcriptome; matrisome

Word count: Abstract: 158

Manuscript: 13.576

\section{Corresponding author:}

Caroline Cheng, Department of Nephrology and Hypertension, Division Internal Medicine and Dermatology, University Medical Center Utrecht. PO Box 85500, 3508 GA Utrecht, the Netherlands. Phone: +31 (0)-88-7557329. Fax: +31 (0)-88-7556283, E-mail: K.L.Cheng-2@umcutrecht.nl 


\section{ABSTRACT}

Dilated cardiomyopathy (DCM) is a relatively common heart muscle disease characterized by the dilation and thinning of the left ventricle accompanied with left ventricular systolic dysfunction. Myocardial fibrosis is a major feature in DCM and therefore it is inevitable that corresponding extracellular matrix (ECM) changes are involved in DCM onset and progression. Increasing our understanding of how ECM adaptations are involved in DCM could be important for the development of future interventions. This review article discusses the molecular adaptations in ECM composition and structure that have been reported in both animal and human studies of DCM. Furthermore, we provide a transcriptome-based catalogue of ECM genes that are associated with DCM, generated by using NCBI Gene Expression Omnibus database sets for DCM. Based on this in silico analysis, many novel ECM components involved in DCM are identified and discussed in this review. With the information gathered, we propose putative pathways of ECM adaptations in onset and progression of DCM. 


\section{Dilated cardiomyopathy}

In the developed world $1-2 \%$ of the population suffers from heart failure, from which approximately half of the patients have systolic heart failure with a reduction in ejection fraction of at least $40 \%$. The underlying cause for systolic heart failure is predominantly ischemic heart disease and dilated cardiomyopathy (DCM). DCM is a relatively common heart muscle disease with a prevalence that increases with age: it is rare in the pediatric population (1-2:100,000), however in adults it has an estimated prevalence of $1: 2500[1,2]$. The disease is characterized by the dilation and thinning of the left ventricle accompanied with left ventricular systolic dysfunction [3, 4]. The diagnosis is primarily based on evidence of dilation and impaired contraction of the left ventricle (left ventricular ejection fraction (LVEF) <45\%) [3-5]. DCM has a poor prognosis with a five-year survival rate of approximately $50 \%$ after diagnosis [6].

The etiology of DCM can be divided into ischemic (50-70\%) and non-ischemic (30-50\%), with the latter phenotype including genetic and acquired causes [7]. In Western countries, $20-50 \%$ of DCM patients have evidence for familial disease. Autosomal dominant is the primary mode of inheritance, although X-linked, recessive and mitochondrial inheritance occur as well. It is important to consider that sporadic DCM cases can also be due to genetic mutations $[4,8]$. Genetic forms of DCM are caused by mutations in cytoskeletal, sacromeric protein, Z-band, nuclear membrane, intercalated disc protein genes etc. $[3,8]$. Recently, up to 110 nuclear genes and 24 mitochondrial DNA genes have been linked to DCM [9]. Regarding the acquired causes of DCM; chronic myocardial inflammation (myocarditis) can lead to DCM at a later stage and is most commonly evoked by infectious triggers. The majority of non-ischemic DCM cases ( $\geq 70 \%$ ) are considered idiopathic: they remain unexplained after a thorough search for primary or secondary causes [7].

Myocardial fibrosis is a major feature in DCM [10-12]. In cardiac tissue, fibrosis has often been described to develop in the setting of an ischemic scar as a result of a myocardial infarction (MI). This reparative response is referred to as replacement fibrosis: dead cardiomyocytes are replaced mainly by collagen. Myocardial fibrosis has also been increasingly recognized in diffuse form in the absence of an ischemic trigger as reported in cardiomyopathies, including DCM $[10,11]$. Here, the cardiac interstitial space expands without cell loss, leaving a patchy fibrotic pattern in the myocardium or large collagen strands in between cardiomyocytes. This type of fibrosis is called reactive or interstitial fibrosis. Myocardial fibrosis is a pathogenic form of extracellular matrix (ECM) remodeling and therefore it is inevitable that corresponding ECM changes are involved in DCM development and progression.

Various animal and human studies have been performed in the last decades trying to elucidate the role of ECM components in DCM and the clinical manifestation of DCM, systolic heart failure (SHF). Studies based on non-ischemic and non-genetic DCM and SHF are summarized and briefly discussed in the following two paragraphs. The third paragraph will discuss the new information gathered from the online NCBI Gene Expression Omnibus (GEO) database sets for DCM. Based on this, we propose 
putative pathways of ECM adaptations in onset and progression of DCM.

\section{Extracellular matrix adaptation in myocardial fibrosis associated with systolic heart failure and dilated cardiomyopathy}

\subsection{Animal studies}

\subsubsection{Collagen and type I/III collagen ratio}

In relation to SHF and DCM, various animal models demonstrated that these diseases are causally associated with changes in ECM composition, structure and function. These changes are summarized in Table 1. Already in 1990, Weber and colleagues [13] performed a histopathological study with dilated LVs of dogs that underwent rapid ventricular pacing. In these tissue samples, disruption by degradation and disappearance of collagen fibers together with interstitial fibrosis in the midwall and epimyocardium was observed. The collagen network in the heart consist mostly of type I and III collagen, that self-organize into fibrils with a diameter ranging from 0.01 to $3 \mu \mathrm{m}$ [14]. These fibrillar collagens aggregate into fibers that are connected through cross-links and form a strong tensile network that tethers cardiomyocytes in support of their alignment. Supported by this typical ECM configuration, the myocyte-generated force can be transduced in all directions. Pro-collagen molecules in their long and rigid triple helix forms are synthesized mostly by fibroblasts and secreted into the interstitial space. Here they will undergo cleavage of their end-terminal pro-peptide sequences to enable collagen fiber formation, since the resultant collagen molecule will be less soluble. This process is crucial in collagen network formation as it initiates self-assembly into collagen fibrils. Quantification of these cleaved terminal peptides can serve to measure the activity of the fibrotic process [15].

The ratio of type III to type I collagen can be considered as an index of myocardial distensibility, since type I is stiffer and provides more tensile strength when compared to type III, that is more elastic. The collagen type I/type III ratio is relatively stable in the healthy myocardium. However, in cardiomyopathies, studies imply that this ratio will shift. Subsequent studies performed by various research groups have analyzed in more detail the role of specific ECM components in DCM development and progression (Table 1). A study of ECM changes in a popular experimental model of DCM, hereditary dilated cardiomyopathy in hamsters (BIO 53.58 hamsters), demonstrated that type III collagen increases significantly at 10 weeks of age, whereas the total collagen content remains stable in control hamsters [16]. In addition, Masutomo and colleagues [17] found a significant increase in the collagen type III:I ratio in the hearts of BIO 53.38 hamsters at 20 and 40 weeks of age, when compared with age-matched control hamsters, further suggesting that the ECM of the affected hamsters was decreased in myocardial stiffness. Collagen crosslinks increased as cardiomyopathy progressed in BIO 53.58 hamsters, suggesting that the formation of crosslinks is activated at the stage of cardiac dilation. The solubility of collagen depends on the amount of 
crosslinks formed. Therefore, this advanced cross-linking in BIO 53.58 hamsters may indicate a progressive decrease in acid solubility of collagen [16]. In contrast, Woodiwiss and colleagues [18] reported a decrease in the ratio of myocardial insoluble to soluble collagen in two different rat models of systolic chamber dysfunction with LV dilation. In one of these models, an increase in the myocardial collagen type I to type III ratio was observed due to an increase in type I collagen, that is again not in line with the findings in the BIO 53.58 hamsters. Adaptation of collagen composition during cardiac LV dilation appears to be either model or species specific and caution should be taken to extrapolate these findings to the human condition.

\subsubsection{Balance in ECM degradation by matrix metalloproteinases and their inhibitors}

The ECM architecture of the cardiac wall is maintained by balanced protein synthesis and degradation. Collagens are synthesized by cardiac fibroblasts, which are in turn activated by different factors in the microenvironment. Potent stimulators of collagen-production by cardiac fibroblasts include growth factors such as transforming growth factor- $\beta$ (TGF- $\beta$ ), nitric oxide and components of the reninangiotensin-aldosterone system (RAAS) [19]. At the other end of the spectrum, ECM degradation is mediated by a family of enzymes called matrix metalloproteinases (MMPs), that are most commonly secreted by fibroblasts among other cells including endothelial cells (ECs), cardiomyocytes and inflammatory cells. The main MMPs present within the cardiovascular tissue are MMP-1, -2 and -9 . MMP-1 is the key enzyme in collagen degradation and facilitates breakdown of the collagen molecule into two fragments. Further degradation steps are carried out by two different enzymes, MMP-2 and 9. In general, MMPs are kept in their latent form by specific inhibitors called tissue inhibitors of MMPs (TIMPs), that colocalize with MMPs throughout the myocardium [20]. Four different TIMPs are known, with TIMP-1 being expressed at low levels in the healthy heart. A fine balance between MMPs and their inhibitors is required to reach equilibrium, and disturbances in their ratios will affect the collagen content and consequently the mechanical properties of the cardiac wall.

MMPs have been demonstrated to play an important role in ECM remodeling in a number of pathological processes associated with cardiac diseases, including DCM (Table 1). Spinale and colleagues [21-24] used the pacing-induced cardiomyopathy pig model to test if LV dilation and remodeling during the progression of congestive heart failure is associated with early changes in MMPs activity and expression. These pigs experience systolic dysfunction and reduced collagen support between adjacent myocytes. Pacing-induced CM is associated with a reduction in the collagen concentration and cross-linking and with an increase the ECM component chondroitin sulfate proteoglycan within the extracellular space. However, a 4-week recovery period results in an increase in the collagen concentration, increased LV stiffness and normal proteoglycan distribution [25, 26]. In subsequent studies, a time-dependent increase was observed in overall MMP activity and protein abundance of MMP-1, 2 and 3 in the pacing group that was accompanied by a decrease in LV myocardial collagen content. These changes co-occurred with the initiation and progression of LV dilation $[21,24]$. More research revealed that chronic inhibition of multiple MMPs in the pacing group attenuated the degree of LV dilation and improved LV ejection performance in comparison with 
untreated rapid pacing pigs [21-23]. Additional studies used the same MMP inhibition therapy in rat and mice models for heart failure: in general, all animals receiving a broad-spectrum inhibitor of MMPs showed diminished ventricular dilation and preservation of systolic function [27-29]. In line with these findings, mice with a Timp-3 deficiency developed spontaneous left-ventricular dilation and contractile dysfunction, coincided with increased MMP-9 activity [30].

Activation of MMPs was also causally related to LV dilation in animal models of SHF. Nishikawa et al. [31] demonstrated that both MMP-2 and -9 activation and protein expression is increased in SHF rats throughout the heart wall. The gene expression of type I collagen, Timp-1 and -2 is significantly enhanced in the SHF model as well when compared with control rats. More evidence for the involvement of MMPs in LV dilation comes from a study that used transgenic mice with cardiac restricted overexpression of TNF $\alpha$. These mice develop progressive LV dilation and structural remodeling from 4 to 12 weeks of age. Sivasubramanian and colleagues [32] showed that during the early phases of LV dilation, the total MMP activity increases significantly and corresponds to a decrease in total myocardial fibrillary collagen content in these mice. However, as the mice age, the total MMP activity significantly decreases and is accompanied by an increase in the total amount of fibrillary collagen. These changes were related to a progressive increase in TIMP-1 levels from 4 to 12 weeks of age. In conclusion, MMPs might be an early event in the development of DCM that co-occurs with LV dilation. This process can be counteracted by mechanisms that activate TIMPs regulation at later stages.

\subsubsection{MMPs and TIMPs in inflammatory DCM}

Myocarditis and subsequent heart damage can ultimately lead to DCM. It is assumed that during the inflammatory phase, ECM remodeling might be triggered causing DCM at a later stage. Three different studies investigated this notion by infecting mice with cardiovirulent coxsackie virus B3 (CVB3), a major cause of myocarditis in humans, and assessing the expression of several MMPs and TIMPs as a measure of ECM remodeling (Table 1). Li and colleagues [33] demonstrated that viral infection causes a significant upregulation of MMP-3 and -9 MRNA and protein along with a downregulation of TIMP-1 and -4 mRNA and TIMP-4 protein. Furthermore, an increase of the degraded soluble fraction of type I collagen was found in these animals. Cheung and co-workers [34] found that already at 9 days post infection (pi), mRNA and protein abundances of MMP-2, -9 and -12 are upregulated, whereas the protein expression of TIMP-3 and -4 are down regulated. During the inflammatory phase (day $9 \mathrm{pi}$ ), the activity of both MMP-2 and -9 is increased as well. Moreover, the murine hearts showed an increase in the total amount of collagen during the acute phase of infection (day 9 pi). In line with the previous studies, Heymans et al. [35] showed an increase in transcript levels of several MMPs, including MMP-2, $-3,-8,-9$ and -13 in mice 7 days after CVB3 infection. Furthermore, the activity and protein level of MMP-9 is significantly enhanced. Interestingly, loss of MMP activity through overexpression of TIMP-1 decreases cardiac inflammation and prevents cardiac dilation at 7 days pi. Besides MMPs, the activity and transcript level of another proteolytic enzyme was found to be enhanced in this study: urokinase-type plasminogen activator (UPA). This enzyme converts 
plasminogen into its active form plasmin, which is responsible for the induction of MMP-9. Absence of UPA reduces the presence of active plasmin in CVB3-infected hearts and correspondingly, a reduction in MMP-9 activity was seen. Furthermore, absence of uPA reduces the inflammation and fibrosis 7 days pi and subsequently protects against cardiac dilation [35]. These findings demonstrate that ECM remodeling is triggered during the inflammatory phase prior to DCM and an early reduction of this process may protect against the dilation.

\subsubsection{Connective tissue growth factor}

Connective tissue growth factor (CTGF) is a glycoprotein that binds the ECM. Szabó and colleagues [36] recently investigated the role of CTGF in regulating the development of cardiac fibrosis during heart failure. Mice were subjected to pressure overload by thoracic aortic constriction (TAC) and received CTGF monoclonal antibodies ( $\mathrm{mAb}$ ) to antagonize CTGF. Animals treated with the target mAb showed improved LV systolic function and reduced LV dilation when compared to untreated mice. Furthermore, CTGF mAb treatment reduces the type I collagen production induced by TAC. In addition, the gene expressions of ECM components Serpin-E1 and Mmp-2 were decreased in CTGF mAb-treated mice.

Studying the effect of preventing LV dilation and preserving normal LVEF during the advancement of heart failure on ECM composition can give useful insights in the involvement of these components in the development of DCM. Aortocaval fistula (ACF) induced LV volume overload leads to progressive LV chamber dilation and systolic dysfunction. Hutchinson and colleagues [37] demonstrated that ACFinduced rats, that underwent a load-reversal procedure, exhibited improved LV chamber dimensions and function. Total collagen content was significantly decreased following ACF, but returns to normal levels after 15 weeks. ACF significantly increases the gene expressions of type I and III collagen and elastin but these were blunted at the reversal stage. Moreover, protein levels of MMP-13 and -14 were increased in ACF-induced rats, but returned to normal values after reversal. In contrast, MMP-7 remained increased after reversal. The reversal procedure caused a decrease of MMP-9 and TIMP-2 protein expression below normal values. These results show that reversal of LV dilation promotes a shift in ECM expression pattern that is associated with heart function normalization.

A summary of the expression profile of ECM components in the different cardiac animal models with LV dilation and systolic dysfunction is provided in Table 1. Only significant and direct changes in ECM components are included.

\subsection{Human studies}

\subsubsection{Collagen and type I/III collagen ratio}

Several human studies have elucidated the role of ECM turnover in the development and progression of SHF and DCM. These changes are summarized in Table 2. Most of the observations were supportive 
of the findings in the animal studies. For example, Gunja-Smith and colleagues were one of the first to provide evidence that DCM is characterized by more matrix deposition due to an increase in the collagen content. The newly produced collagen is deficient in forming stable cross-links and may therefore contribute to the dilation of the ventricular wall $[38,39]$. Numerous human studies reported a similar increase in total collagen content, that mainly consists of collagen type I and III with an increase in the collagen type I/type III ratio. In general, collagen type I provides more tensile strength and stiffness compared to collagen type III [40-47]. Besides type I and III collagen, other collagens, such as type IV and VI are present in minor quantities in the healthy myocardium. However, in DCM hearts the enlargement of the extracellular space is associated with abundant collagen VI $[47,48]$. In concordance with the results obtained from animal studies, DCM is characterized by an increase in both collagen type I and III. In addition, all human studies reported a clear increase in the collagen type I to type III ratio, that may contribute to the DCM phenotype due to an increase in stiffness and decrease in elasticity of the cardiac wall. Furthermore, Sivakumar et al. [46] found that both transcriptional and translational levels of lysyl oxidase (LOX), a key ECM enzyme catalyzing crosslinks in collagen and elastin, were significantly upregulated in DCM hearts.

\subsubsection{Balance in ECM turnover mediated by MMPs and TIMPS}

DCM is also characterized by enhanced matrix turnover (Table 2). Gunja-Smith and colleagues [38, 39] were one of the first to demonstrate that this shift is caused by an increase in MMP activity. In more detail, both active and latent MMP-1 and -8 levels were found to be higher in DCM hearts, whereas the expression of their inhibitor, TIMP-1, felt to negligible levels. Several additional studies have been performed on the role of MMPs in DCM, in which an overall increase in total MMP activity was observed [49]. Tyagi et al. [50, 51] demonstrated that MMPs are present in the latent form in the normal myocardium, but are activated in DCM. In more detail, MMP-1 expression was found to be induced in the DCM heart on both mRNA, protein and activity level when compared to normal tissue. Furthermore, TIMP-1 is repressed in DCM. Rouet-Benzineb et al. [52] found that besides TIMP-1; TIMP-2, -3 and -4 were downregulated in ventricle samples from DCM patients when compared to healthy controls. However, the activity of MMP-2 and -9 remains unchanged. In contrast, two studies reported that MMP-1 levels were decreased in DCM $[53,54]$. Several studies found MMP-2, -3 and -9 levels to be increased in DCM myocardial tissue when compared to non-failing hearts. However, in contrast with previous results, a rise in TIMP-1, -2 and -3 levels was also detected $[46,53,54]$. In addition, MMP-14 was found to be also increased in DCM hearts [53]. An increase in MMP-1 and -9 protein expression, with an increase in the MMP-1 to TIMP-1 ratio was observed in DCM patients by Klotz and colleagues [42]. These mixed findings suggest that a complex interplay between MMPs and their inhibitors TIMPs exists in the DCM heart.

\subsubsection{Evidence provided by serological markers}

More evidence for an enhanced matrix turnover in DCM comes from studies performed on serological markers of collagen metabolism (Table 2). It has to be noted, that evidence based on serological 
markers should be considered with caution, since the difference in serum concentration can also be the result of secondary effects due to forward failure that decreases the blood flow to various organs. Klappacher and colleagues [55] demonstrated that patients with DCM have higher serum concentrations of two collagen cleavage products: procollagen type III aminoterminal peptide (PIIINP) and type I collagen carboxyterminal telopeptide (ICTP) compared to healthy controls. In addition, the authors reported that serum PIIINP and ICTP positively correlates with their tissue analogues, myocardial collagen type III and I, making these results more reliable. Schwartzkopff and colleagues [56] showed an increase in serum ICTP, a marker for collagen type I degradation, but no increase was reported for procollagen type I carboxyterminal propeptide (PICP), a marker for collagen type I synthesis. They also demonstrated an elevation in serum MMP-1 and TIMP-1 concentrations in DCM patients and a higher MMP-1/TIMP-1 ratio than in control subjects. Other studies have investigated the serum concentrations of MMPs and their inhibitors in patients with SHF. According to Yan and colleagues [57], patients with chronic SHF (LVEF <40\%) have higher plasma levels of MMP-2 compared to baseline at 43 weeks of follow-up. MMP-9 and TIMP-1 plasma levels did not change significantly overall in the study population. However, elevated MMP-9 levels correlates with lower LVEF and deterioration of LV function. Results obtained by Jordán et al. [58] demonstrated that patients with systolic dysfunction (LVEF $<50 \%$ ) have lower levels of MMP-1 and higher levels of TIMP1 in their serum. This results in a higher TIMP-1/MMP-1 ratio than in healthy controls. In yet another study population of patients with SHF (LVEF $<45 \%$ ), serum MMP-1 and -2 levels were found to be significantly upregulated in SHF patients on admission when compared to healthy subjects. Only MMP1 levels remained high at discharge [59].

In addition to collagen and MMPs, other extracellular matrix components are differentially expressed in DCM. Klappacher and colleagues [55] demonstrated that patients with DCM have higher serum concentrations of basement membrane laminin when compared to healthy controls, that positively correlates with myocardial laminin content. Serum tenascin C (TNC), a glycoprotein that is specifically expressed at high levels during embryogenesis, but not in the adult heart, was found to be increased in DCM patients compared to healthy controls. Moreover, a significantly negative correlation was found between TNC levels and LVEF [60]. TNC is mostly deposited along the margin of the replacement fibrotic lesions found in DCM hearts, implying that the fibrotic change is a continuous process [61].

Besides differences between healthy and DCM hearts, ECM components also differ in distribution within the diseased myocardium. The volume densities of fibronectin, collagen type I and IV were reported to be higher in the left ventricle than in the right ventricle of DCM hearts [62]. Another group observed fibronectin in both the endomysium and perimysium of DCM heart biopsy specimens, coexistent with collagen type I and III. Laminin was also observed in the endomysium and was localized together with collagen type IV. In addition, fibronectin and collagen type I, III and IV are nonhomogenously distributed in the replacement fibrotic lesions [63]. Furthermore, pediatric DCM is 
characterized by a specific profile compared with adult DCM: MMP-8, MMP-9, TMP-1 and TMP-2 levels are increased in pediatric DCM, whereas MMP-3, MMP-7 and TIMP-3 levels are decreased [64].

A summary of the expression profile of ECM components in the human DCM and SHF populations is provided in Table 2. Only significant changes in ECM components are included and indirect but significant serum changes are also mentioned.

It has to be noted that there is a discrepancy in findings between animal and human studies, in particular differences in MMP expression. An almost unanimous enhanced MMP expression is seen in the animal studies, with the exception of MMP-14, whereas in the human studies the overall expression pattern found is contradicting. Animal models provide the opportunity to study ECM changes in a more controlled environment and allow for the assessment of more subtle changes. Even though the findings obtained from animal models are more consistent, the question remains if animals are reliable enough in predicting the humane response. Only one animal model mentioned in this review, the BIO 53.58 hamsters, resembles the human situation in terms of developing DCM over time without intervention. In all the other models, the DCM phenotype had to be induced experimentally. The reason for the opposing findings in MMP expression within the human studies could be explained by the fact that these studies cannot be controlled on the same level as animal studies. Factors such as timing, heterogeneity of the patient population, sample size, biopsy location and the fact that various studies investigate only mRNA or protein levels of relevant factors among other issues contribute to the rise of contradicting results. It is also important to point out that the majority of studies investigate mRNA and/or protein levels of the MMP/TIMP system, whereas the overall net enzymatic activity is the determining factor in terms of ECM remodeling. The amount of contradictory data in the field highlights the need for additional studies.

\section{Novel extracellular matrix changes identified in dilated cardiomyopathy by GEO datasets analysis}

The human matrisome is composed of an ensemble of ECM and ECM-associated proteins, of which some have been suggested to play a role in DCM onset and progression, as discussed in previous paragraphs. We analyzed specific gene expression datasets that are publicly available on the NCBI's GEO database (http://www.ncbi.nlm.nih.gov/sites/GDSbrowser) to find novel ECM components that are altered in cardiac expression by DCM and might be involved in disease development. We selected three datasets from studies that included human DCM and non-failing heart samples in their study design, see supplemental Figure 1 for an overview of the pipeline used for dataset selection. Two of the three selected studies obtained their tissue biopsies from the left ventricle and the third used septal myocardial tissue. Other characteristics of the patient subset used in each study are 
summarized in Table 3. NCBI's GEO2R tool (www.ncbi.nlm.nih.gov/geo/geo2r/) was used in order to identify genes that are significantly differentially expressed between the DCM and healthy control groups. Datasets matching the earlier described criteria were downloaded (Supplementary Figure 1). Each raw dataset was sorted by R script provided by GEO2R program. Gene expression was analyzed as following: (1) P-value below 0.05 was used to filter genes that reached significant level; (2) Selected genes with logFC value above 0 represented increased expressions, and those with logFC value below 0 represented decreased expressions. (3) Genes with significantly increased and/or decreased expression were mapped among all matrisome subgroups. The list of ECM genes associated with DCM were obtained from the resulting expression data after filtering with an in silico matrisome dataset (http://matrisomeproject.mit.edu/). This dataset contains all the components of the human matrisome based on characterization and bioinformatic prediction, subdivided into two main categories: the core matrisome and ECM-associated components [65, 66]. The core matrisome consist of glycoproteins, collagens and proteoglycans, whereas the categories ECM-affiliated, ECM regulators and secreted factors represent the ECM-associated group. The resulting expression datasets are summarized in Table 4 and 5 and Figure 1.

Many genes are differentially expressed between the DCM and healthy control groups in all three datasets (Table 4 and 5 ). From these, $\pm 5 \%$ can be assigned to the human matrisome. From the genes that are increased in DCM hearts, half is represented by core matrisome components. Further classification reveals that most of these genes can be assigned to the glycoprotein family. Collagens and proteoglycans are present as well, but in lower quantities. The remaining genes increased in DCM based on the GEO analysis are represented by matrisome associated components, with approximately equal distribution between the three subgroups. A different pattern is observed within the group of down regulated matrisome genes. Less genes are represented by core matrisome components, ranging from $8-31 \%$ between the different datasets. Nevertheless, as with the upregulated genes, most of the core genes are classified as glycoproteins. In contrast with the other two sets, in dataset GSE42955, none of the downregulated genes are neither collagens nor proteoglycans. The majority of downregulated genes can be classified as matrisome associated, with the secreted factors being the most represented subgroup. All the genes that are differentially expressed in the three datasets are listed in the supplemental data (Supplementary Tables 1-4 and supplementary Figures 2).

To look in more detail at the differential gene expression data obtained from the GEO analysis, a heat map was constructed (Figure 1). Only genes are included that are significantly up- or downregulated in at least two datasets. Conform the literature for both animal and human studies summarized in the previous sections, collagen type I and III are upregulated in DCM hearts based on our GEO analysis. In addition, the glycoproteins elastin and laminin are upregulated as well, the latter for the subtypes $\alpha 2$ and $\beta 1$. On the other hand, UPA is found to be upregulated in CVB3-infected hearts, however in our analysis the expression is found to be reduced in DCM hearts. In addition, tenascin C, lysyl oxidase and chondroitin sulfate proteoglycan are mentioned in the literature as ECM components with increased expression in DCM, but are not significantly upregulated in our analysis. Strikingly, neither MMPs nor TIMPs are found as differentially expressed, even though many studies 
have provided evidence for their involvement in DCM pathology. An explanation for missing these important ECM regulators in the GEO analysis could be that ECM degradation through MMP activity is a cyclic and transient process. When sampling at specific (later) time points, lower levels of MMPs and TIMPs may present within the biopsies.

\subsection{ECM regulators}

Within the ECM regulators group, we find ECM components that play a role in ECM turn-over and stabilization that are differentially expressed (Figure 1 and 2). For example, HTRA1 is a highly conserved serine protease that degrades numerous extracellular matrix proteins, including collagen and fibronectin. An upregulation in HTRA1 is observed in our analysis, hinting at more matrix degradation in DCM. Subsequent fibronectin cleavage products can promote transcriptional activation of MMPs, that will additionally contribute to matrix degradation. Furthermore, HTRA1 has also the ability to sequester or proteolyse TGF- $\beta$. Since TGF- $\beta$ induces fibroblasts to synthesize ECM components, degradation of TGF- $\beta$ will result in more matrix remodeling [67]. In addition, two members of the proprotein convertase family, PCSK5 and PCSK6, show a higher expression in DCM. These serine proteinases activate other proteins, thereby regulating their functional activity. The processing of proMMP-14 as well as its proteolytic activity has been shown to be catalyzed by PCSK5, thereby indirectly inducing more breakdown of the extracellular matrix [68].

However, many more ECM regulators found in our analysis contribute to ECM synthesis and stabilization (Figure 1 and 2). For instance, P4HA2 expression is upregulated in our analysis, that encodes a component of prolyl 4-hydroxylase. This is a key enzyme in collagen synthesis and is composed of two $\alpha$ and two $\beta$ subunits. The $\alpha 2$ subunit composes a major part of the catalytic site of the active enzyme. In collagen and related proteins, prolyl 4-hydroxylase catalyzes the formation of 4hydroxyproline residues that are essential for stabilization and proper three-dimensional folding of newly synthesized collagen [69]. The expression of PLOD1, an enzyme that catalyzes the hydroxylation of lysine in collagens and related proteins, is also upregulated. The resultant hydroxylysine groups serve as attachment sites and are therefore critical for the formation and stability of intermolecular collagen cross-links. In addition, ADAMTSL5 and ITIH5 are induced during DCM and can contribute to ECM strengthening. ADAMTSL5 can bind to both fibrillin-1 and -2 and is thought to promote fibrillin microfibril formation [70, 71]. The ITIH5 gene encodes a heavy chain component of one of the inter- $\alpha$-trypsin inhibitor family members, that are thought to be involved in extracellular matrix stabilization [72]. Additional contribution to preserve the ECM may come from the downregulation of the genes A2M, ADAMTS9, CD109 and PLAU, as seen in our analysis. The A2M gene encodes a protease inhibitor that inhibits many proteases, including collagenases (e.g. MMP-2 and -9) [73]. ADAMTS9 is a member of the ADAMTS protein family, that is associated with the cleavage of two large aggregating proteoglycans, aggrecan and versican, resulting in less ECM degradation [74]. CD109 has been identified as a negative regulator of TGF- $\beta$ signaling. It increases binding of TGF- $\beta$ to its receptors, enhances their internalization and facilitates their degradation [75]. 
Thus, downregulation of CD109 will result in more TGF- $\beta$ signaling. PLAU encodes for the enzyme uPA that is part of the extracellular protease system. It converts plasminogen into plasmin, that is an important fibrinolytic protease with a wide range of substrates. Plasmin can directly degrade ECM components, such as fibronectin, that binds to cell surfaces and various compounds including collagen and fibrin [76]. In addition, plasmin is needed to activate a wide number of MMPs, including MMP-3, $9-12$ and -13 [77]. Therefore, downregulation of PLAU will result in less active plasmin and subsequent less degradation of ECM components and fewer active MMPs available to degrade collagens. This is in line with the result mentioned in the animal data section that loss of uPA protects against cardiac dilation during CVB3-induced myocarditis [35].

To conclude, in the DCM group, more ECM regulators are involved in ECM synthesis and stabilization compared to ECM degradation, therefore the balance in DCM may be shifted towards a stiffer and denser ECM (Figure 2).

\subsection{Secreted factors}

Besides ECM regulators, the secreted factors compose another major group within the heat map (Figure 1). Within this group, two members of the fibroblast growth factor family are presented. These components are essential regulators of the proliferation, chemotaxis and survival of numerous structural cells, including fibroblasts. During normal tissue repair, fibroblasts proliferate and are recruited into the lesion, where they remodel the ECM by producing, adhering to and contracting the ECM. Eventually the fibroblasts disappear due to apoptosis and normal tissue function is restored. Excessive fibrosis is the result of fibroblasts persisting in the wounded area, resulting in scarring [78]. Myocardial fibrosis is a major feature in DCM and excessive fibroblast activation and survival due to the upregulation of fibrogenic components might contribute to the formation of scar tissue in the DCM heart (Figure 1 and 3). FGF1 is upregulated in our GEO analysis and it was already known in 1974 that FGF1 stimulates fibroblasts proliferation [79]. Intracellular FGF1 has the ability to inhibit both p53-dependent apoptosis and cell growth arrest in rat embryonic fibroblasts by decreasing the stability of p53 and through modification of the transactivation properties of p53 [80]. FGF7 expression is reduced in DCM, however, this protein is only mitogenic for epithelial cells and not for fibroblasts [81]. More secreted factors with a high gene expression in our analysis are found to be positively linked to fibroblast activity. For example, PTN is another growth factor that is recognized as a mitogen towards fibroblast [82]. In addition, PTN can inhibit apoptosis of NIH3T3 fibroblasts through activation of the MAPK pathway [83]. We also see an induction of FSTL3 expression in our GEO analysis, that is a potent inhibitor of activin signaling and angiogenesis. It has been shown that production of FSTL3 by cardiomyocytes contributes to the paracrine activation of cardiac fibroblasts. An increased gene expression will promote proliferation, increase collagen production and induce changes in cell adhesion [84]. Also, cultured human fibroblasts express constitutive IL-16 and CRLF1 mRNA. Both these molecules are found to be upregulated in DCM, giving an indirect indication of induced fibroblast activity $[85,86]$. 
On the contrary, other factors found in our GEO analysis that are associated with fibroblast activity are downregulated, which could contribute to an antifibrogenic effect (Figure 1 and 3). Two of these components are CCL2 and $I L-6$. CCL2 can induce IL-6, that in turn can mediate fibroblast survival by inhibiting apoptosis via the ERK1/2 signaling pathway [87]. The expression of $C C L 11$, a small cytokine that can modulate fibroblast activity by increasing their proliferation, chemotaxis, collagen synthesis and MMP-2 activity, and FIGF, a growth factor that is able to stimulate mitogenic activity and induce morphological alterations in fibroblasts, are both reduced $[88,89]$. Besides FGFs, EGF has also been implicated as a potent mitogenic factor for numerous cell types, including fibroblast. It has been found by several studies using fibroblast in culture that EGF can stimulate fibroblast migration and proliferation and can also promote fibroblast contractility and motility via a protein kinase $C$ deltadependent pathway [90-93]. Furthermore, the EGF receptor is required for MMP expression and collagen contraction in fibroblasts through the MAPK and AP-1 pathways [94]. EGF is also part of the fibroblast growth factor receptor signaling pathway, for it can upregulate TGF- $\beta$ receptor II in human fibroblasts via the p38 mitogen-activated protein kinase pathway [95]. TGF- $\beta$ induces fibroblasts to contract and synthesize ECM and is considered to be a key player in the fibrotic response [96]. Altogether, a downregulation of $E G F$ as found in our analysis will most likely have a negative impact on fibroblast activity. Lastly, CCL8 and FGF7, a small cytokine and growth factor respectively, can be produced by fibroblasts itself. Since the gene expression of both is reduced in our analysis, it might indirectly indicate fibroblast suppression $[81,97]$.

In summary, there is no clear pattern found within the secreted factors in relation with fibroblasts. Instead, it seems that a balance between fibroblast activation and suppression is present in the DCM samples (Figure 3). Different stadia of fibrosis are intermixed in tissue biopsies taken from the DCM heart. This could explain the diverse signals seen within this group. In addition, the suppression of some fibrogenic factors might also be the result of a negative feedback mechanism to prevent excessive fibroblast growth and activation. More research is needed to fully understand the involvement of these factors in fibroblast regulation during DCM onset and progression.

\subsection{Collagens}

We identified highly interesting targets within the matrisome associated group, however, many additional interesting targets were found among the core components, such as various collagen subtypes (Figure 1). Collagens are the most abundant core component in the extracellular matrix that typically contain a characteristic triple helical conformation. The vertebrate collagen family includes more than 40 genes that code for collagen a chains, which can form at least 28 different collagen subtypes [98]. Fibrillar collagens are the main subgroup in vertebrates, where they fulfill a structural role by contributing to the molecular organization, mechanical properties and shape of tissues. In concordance with the extensive literature on fibrillar collagen involvement in DCM, collagen type I and III are significantly upregulated in DCM according to our GEO dataset analysis. These two collagen subtypes are found throughout the body and are known to be synthesized in response to injury. 
Additionally, we find a novel fibrillar collagen enhanced in DCM, namely collagen type V. Only two combinations of a chains can produce this low abundance fibrillar collagen and both genes, COL5A1 and COL5A2, are upregulated. Collagen type $\mathrm{V}$ is found in tissues containing collagen type $\mathrm{I}$ and it appears to have an essential role in initiating collagen fibril assembly [99]. Therefore, the enhanced synthesis and deposition of collagen I seen in the DCM heart might be directly associated with excessive collagen $\mathrm{V}$ production. Furthermore, mutations in these two genes are associated with the Ehlers-Danlos syndrome (EDS). This disorder includes a vascular type characterized by a fragile vasculature with a high risk of lethal vascular events, such as arterial rupture [100], indicating that collagen type $\mathrm{V}$ might be important for vascular stability.

In addition to a novel fibrillar collagen, we find four additional collagen subtypes enhanced in DCM. Two of them, collagen type XIV and XXI, belong to a subgroup of fibril-associated collagens with interrupted triple helices (FACITs) that do not form fibrils by themselves. Instead, these collagens are considered regulators of fibrillogenesis, since they have specific regions designed for the adhesion and interaction with fibrils [101]. Collagen XIV interacts with collagen type I and is found to be highly expressed in areas of high mechanical stress, such as the cardiac muscle [102]. Similarly, collagen type XXI is present in tissues expressing a muscle phenotype enriched with collagen type I. In more detail, high expression of COL21A1 is found within the heart, with the left ventricle expressing lower levels compared to the right ventricle, apex and septum [103]. FACITs are required for the assembly of a tightly regulated collagen network in the heart. In line with this statement, Col14a1-/- mice experience diminished cardiac function, including dilation of the heart and reduced ejection fraction, indicating the importance of collagen type XIV for the structural integrity of the myocardium [104]. In contrast to these results obtained using a murine model, our data indicates that during the progression of DCM in humans, COL14A1 is enhanced. An explanation might be that during the later phases of the disease, a surplus of adhesive collagens is produced as an attempt to strengthen the dilated left ventricle, mostly subtypes that interact with collagen type I.

Besides collagens involved in fibril formation and interactions, additional subgroups of non-fibrillar collagens have been described. Among these are collagens involved in the formation of twodimensional sheets that give structure to protein membranes surrounding tissues. An example of such a membrane is the basement membrane and one of its major constituents is collagen type IV. Two of the six human genes associated with it are upregulated in DCM according to our analysis; COL4A1 and COL4A2. Mice with a Col4a1 missense mutation experience defects in vascular function, including focal detachment of the endothelium, and low blood pressure [105], showing the importance of collagen type IV for the stability of vascular structures during mechanical demand. Furthermore, mutations in both genes have been linked to small-vessel disease and hemorrhagic stroke [106, 107]. Collagen type VIII is another example of a collagen that forms two-dimensional sheets. It is an important component of the subendothelium and it is expressed by ECs and vascular smooth muscle cells (vSMCs) as a heterotrimer composed of a1 and a2 chains. In our GEO dataset analysis, COL8A1 is found to be upregulated in DCM. Collagen type VIII is necessary for the migration and growth of 
vSMCs [108], hinting towards a potential role of this collagen in the maintenance of vessel wall structure and integrity.

In conclusion, besides the well-described collagen I and III, the expression of several other collagen family members is found to be enhanced in DCM, including both fibrillar and non-fibrillar collagens. It is interesting to speculate that this unanimous enhancement of collagen expression is most likely a joined protective response towards the ventricular dilation in other to maintain proper mechanical stability and functionality of the myocardium.

\subsection{Glycoproteins}

Besides collagens, other proteins within the extracellular matrix are considered fibrous proteins, including elastins, fibronectins and laminins. These proteins belong to the family of glycoproteins that composes a major part of the core matrisome and our heatmap as well (Figure 1). Tissues that undergo substantial stretching and bending contain a high amount of the structural glycoprotein elastin, which arranges in bundles called elastic fibers. Particularly the walls of large arteries are rich in elastic fibers allowing them to stretch in response to each pulse and resume their shape afterwards. In addition to structural glycoproteins, specialized glycoproteins are present in the ECM that have an important adhesive role, such as fibronectin and laminin. Both are fibrous glycoproteins that attach cells to the ECM. In more detail, fibronectin exist as a protein dimer that binds both cell-surface receptors called integrins and a broad variety of matrix components, except collagen type IV. Instead, type IV matrices contain laminins as adhesive proteins. All isoforms of fibronectin arise by alternative RNA splicing of the pre-mRNA encoded by a single gene, FN1 [109]. This gene is upregulated in DCM hearts based on our GEO dataset analysis. Conform the literature summarized in the previous sections, the genes encoding elastin and laminin (ELN and LAM, respectively) are upregulated as well, the latter for the subtypes $a 2$ and $\beta 1$. Loss of functionality caused by the dilation and increased stiffness through enhanced collagen deposition might be compensated by an increase in these structural and adhesive glycoproteins.

Fibrous ECM components such as fibronectin are necessary for the incorporation of the latent TGF$\beta 1$ complex into the matrix. The healthy myocardium contains an inactive TGF- $\beta 1$ pool awaiting for activation upon injury or overload. The major effects of TGF- $\beta$ pathway activation include inhibition of proliferation and enhancement of ECM production [110]. Storage of latent TGF- $\beta 1$ requires the presence of latent transforming growth factor-beta-binding proteins (LTBPs) that covalently bind the complex to the matrix. Fibronectin provides a scaffold for LTBP1 in particular, which is an LTPB known to be involved in vascular remodeling [111-113]. LTBP1 is upregulated in our GEO dataset analysis together with FN1. We find LTBP2, the largest member of the LTPB family, to be highly expressed in DCM as well. TGF- $\beta$ is a potent regulator of ECM formation and its deposition seems to be enhanced in DCM hearts.

Evidence for fibronectin accumulation in the damaged heart is found in the early phase post infarction, where it is expected to stimulate adhesion and proliferation of progenitor cells [114, 115]. Furthermore, regulators of fibronectin are also upregulated according to our analysis, hinting towards 
promoted cell adhesion in DCM (Figure 1 and 4). CTGF is a fibrogenic protein which expression is induced by TGF- $\beta$ in various cell types including cardiac fibroblasts and myocytes. By mediating downstream events of the TGF- $\beta$ signaling pathway, CTGF induces the secretion of fibronectin and mediates its matrix deposition by controlling integrin expression $[116,117]$. Another regulator, DPT, interacts with fibronectin and enhances its fibril formation. It is suggested that DPT has an important role in wound healing by inducing cardiac fibroblast adhesion and migration via integrins [118, 119]. More evidence comes from the upregulation of SRPX2 and NDNF. It is demonstrated in vitro that SRPX2 enhances cellular migration and adhesion by increasing the phosphorylation of focal adhesion kinase (FAK), a cytoplasmic protein needed for the activation of integrin related signaling pathways [120]. NDNF is a secretory protein that has fibronectin type III domains. This allows for the speculation that NDNF can modulate integrin-dependent signals. In line with this, Ohasi et al. [121] revealed that NDNF promotes EC function and survival by activating the integrin a5 $\beta 3$-associated pathway.

On the contrary, several components found in our GEO analysis are associated with negative regulation of cell adhesion (Figure 1 and 4). TSP2 has not been extensively studied, however because of its similarity to family-member TSP1; it is believed that it can interact with the same targets including fibronectin [122]. TSP2 inhibits the formation of focal adhesions in ECs in vitro [123] and TSP2-null fibroblasts showed a marked defect in attachment to fibronectin and a reduction in cell spreading [124]. Therefore, the upregulation of TSP2 seen in our analysis will probably have a negative effect on cell adhesion. Furthermore, IGFBP3 associates with the cell binding domain of fibronectin. This allows for the speculation that an upregulation of IGFBP3 as seen in our analysis has the potential to displace cells from the matrix [125]. Downregulation of CRIM1 as seen within our analysis most likely has a negative impact on cell adhesion, since it is demonstrated that silencing CRIM1 expression decreases cell migration and adhesion in vitro [126].

To conclude, the increase of the adhesive glycoproteins fibronectin and laminin in DCM indicates that the response towards the dilation might include enhanced cell adhesion activities and increased deposition of latent TGF- $\beta 1$ into the matrix. Further evidence for enhanced cell adhesion comes from the upregulation of several glycoproteins regulating fibronectin deposition and integrin signaling. The expression pattern of other factors indicate that cell displacement activities are present in de DCM heart as well, possibly functioning as a negative feedback mechanism to prevent excessive cell adhesion (Figure 4). More research is needed to fully understand the involvement of these factors during DCM onset and progression.

\section{Conclusion}

It is inevitable that ECM changes are involved in the development and progression of DCM, since myocardial fibrosis is a major feature of this disease. In this review, we have provided literature 
sources for the involvement of diverse ECM components in non-ischemic and non-genetic DCM and SHF. In both animal and human studies, a consistent increase in collagen type I and III synthesis and deposition has been described, with an increase in the collagen type I/type III ratio. Collagen type I provides more tensile strength, resulting in a stiffer matrix. This excessive production of collagen is most likely an effort to strengthen the heart wall during the progression of DCM. Besides collagen, more core matrix components are abundant within the DCM heart, including elastin, laminin and tenascin C. Furthermore, a complex interplay between various MMPs and TIMPs arises in the DCM heart that includes the main MMPs present within the cardiovascular tissue (MMP-1, 2 and 9) and all four known TIMPs. Based on the results from the animal studies, the enhanced MMP activity and protein abundance might be an early event in DCM that co-occurs with the initiation of LV dilation. Progression of the disease is characterized by a fall in MMPs, due to an increase in TIMPs.

Increasing our understanding of the involvement of the ECM in the onset and progression of DCM is important for the development of future interventions. Here, the focus should not merely lie on the core matrisome components, but on the ECM associated factors as well, that provide biochemical support to the surrounding cells and thus can regulate many biological processes. Our GEO dataset analysis provides a catalogue of ECM genes that might be involved in the development and progression of DCM. These components have the potential in becoming future candidates for therapeutic targets or prognostic markers. Within the matrisome associated components, a considerable part is represented by ECM regulators and secreted factors. Excessive ECM synthesis and stabilization is most likely the joint outcome from the ECM regulators, either by stimulating fibril construction with the formation of extra protein residues and cross-links or by inhibiting proteases and increasing TGF- $\beta$ signaling. Together with the increase in collagen I and III already described in the literature and the enhanced expression of additional fibrillar and non-fibrillar collagens found with our GEO datasets analysis, these results indicate that strengthening the heart wall through ECM remodeling is a major feature of DCM. Furthermore, a wide array of secreted factors is found and their combined effect will likely lead to altered fibroblast activity. Many of these molecules are involved in fibroblast proliferation, apoptosis, migration and the production of ECM components among others. More research is needed to fully understand the involvement of these factors in fibroblast regulation. Finally, within the core components, the increase of the adhesive glycoprotein fibronectin and several regulators of its deposition and associated signaling pathway indicate that the response towards the dilation might include enhanced cell adhesion activities.

This transcriptome-based catalogue of novel ECM components expands the fundamental knowledge about the involvement of ECM remodeling in DCM. However, more research is required before any of these components can be considered as a prognostic marker or as a target for treatment. The function of these potential targets needs to be validated by using in vitro assays and assessing the causality with animal models is recommended to further elucidate the added value of these newly identified ECM components. 


\section{Conflict of interest}

None

\section{Acknowledgements}

The work was supported by Netherlands Foundation for Cardiovascular Excellence [to C.C], two NWO VIDI grants [no. 91714302 to C.C., and no. 016096359 to M.V.], the Erasmus MC fellowship grant [to C.C.], the RM fellowship grant of the UMC Utrecht [to C.C.] and the Netherlands Cardiovascular Research Initiative: An initiative with support of the Dutch Heart Foundation [CVON2014-11 RECONNECT; to C.C., M.V.]. 


\section{References}

[1] B.J. Maron, J.A. Towbin, G. Thiene, et al., Contemporary definitions and classification of the cardiomyopathies: An american heart association scientific statement from the council on clinical cardiology, heart failure and transplantation committee; quality of care and outcomes research and functional genomics and translational biology interdisciplinary working groups; and council on epidemiology and prevention, Circulation 113 (2006) 1807-1816.

[2] J.D. Wilkinson, D.C. Landy, S.D. Colan, et al., The pediatric cardiomyopathy registry and heart failure: Key results from the first 15 years, Heart Fail Clin 6 (2010) 401-413.

[3] P. Elliott, B. Andersson, E. Arbustini, et al., Classification of the cardiomyopathies: A position statement from the european society of cardiology working group on myocardial and pericardial diseases, Eur Heart J 29 (2008) 270-276.

[4] P. Rubis, The diagnostic work up of genetic and inflammatory dilated cardiomyopathy, E-journal of Cardiology Practice 13 (2015).

[5] D.M. Gopal, F. Sam, New and emerging biomarkers in left ventricular systolic dysfunction--insight into dilated cardiomyopathy, J Cardiovasc Transl Res 6 (2013) 516-527.

[6] V.V. Michels, D.J. Driscoll, F.A. Miller, et al., Progression of familial and non-familial dilated cardiomyopathy: Long term follow up, Heart 89 (2003) 757-761.

[7] R.E. Hershberger, D.J. Hedges, A. Morales, Dilated cardiomyopathy: The complexity of a diverse genetic architecture, Nat Rev Cardiol 10 (2013) 531-547.

[8] E.M. McNally, J.R. Golbus, M.J. Puckelwartz, Genetic mutations and mechanisms in dilated cardiomyopathy, ] Clin Invest 123 (2013) 19-26.

[9] M. Harakalova, G. Kummeling, A. Sammani, et al., A systematic analysis of genetic dilated cardiomyopathy reveals numerous ubiquitously expressed and muscle-specific genes, Eur J Heart Fail 17 (2015) 484-493.

[10] A. Brooks, V. Schinde, A.C. Bateman, P.J. Gallagher, Interstitial fibrosis in the dilated non-ischaemic myocardium, Heart 89 (2003) 1255-1256.

[11] M.A. Heneghan, D. Malone, P.A. Dervan, Myocardial collagen network in dilated cardiomyopathy. Morphometry and scanning electron microscopy study, Ir J Med Sci 160 (1991) 399-401.

[12] D.V. Unverferth, P.B. Baker, S.E. Swift, et al., Extent of myocardial fibrosis and cellular hypertrophy in dilated cardiomyopathy, Am J Cardiol 57 (1986) 816-820.

[13] K.T. Weber, R. Pick, M.A. Silver, et al., Fibrillar collagen and remodeling of dilated canine left ventricle, Circulation 82 (1990) 1387-1401.

[14] J.B. Caulfield, P.E. Wolkowicz, Myocardial connective tissue alterations, Toxicol Pathol 18 (1990) 488-496.

[15] C. Jellis, J. Martin, J. Narula, T.H. Marwick, Assessment of nonischemic myocardial fibrosis, J Am Coll Cardiol 56 (2010) 89-97.

[16] H. Okada, H. Kawaguchi, T. Kudo, et al., Alteration of extracellular matrix in dilated cardiomyopathic hamster heart, Mol Cell Biochem 156 (1996) 9-15.

[17] K. Masutomo, N. Makino, T. Maruyama, T. Shimada, T. Yanaga, Effects of enalapril on the collagen matrix in cardiomyopathic syrian hamsters (bio 14.6 and 53.58), Jpn Circ J 60 (1996) 50-61.

[18] A.J. Woodiwiss, O.J. Tsotetsi, S. Sprott, et al., Reduction in myocardial collagen cross-linking parallels left ventricular dilatation in rat models of systolic chamber dysfunction, Circulation 103 (2001) 155-160.

[19] V.I. Kapelko, Extracellular matrix alterations in cardiomyopathy: The possible crucial role in the dilative form, Exp Clin Cardiol 6 (2001) 41-49. 
[20] S.C. Tyagi, S.G. Kumar, J. Banks, W. Fortson, Co-expression of tissue inhibitor and matrix metalloproteinase in myocardium, J Mol Cell Cardiol 27 (1995) 2177-2189.

[21] M.L. Coker, C.V. Thomas, M.J. Clair, et al., Myocardial matrix metalloproteinase activity and abundance with congestive heart failure, Am J Physiol 274 (1998) H1516-1523.

[22] J.H. McElmurray, 3rd, R. Mukherjee, R.B. New, et al., Angiotensin-converting enzyme and matrix metalloproteinase inhibition with developing heart failure: Comparative effects on left ventricular function and geometry, J Pharmacol Exp Ther 291 (1999) 799-811.

[23] F.G. Spinale, M.L. Coker, S.R. Krombach, et al., Matrix metalloproteinase inhibition during the development of congestive heart failure : Effects on left ventricular dimensions and function, Circ Res 85 (1999) 364-376.

[24] F.G. Spinale, M.L. Coker, C.V. Thomas, J.D. Walker, R. Mukherjee, L. Hebbar, Time-dependent changes in matrix metalloproteinase activity and expression during the progression of congestive heart failure: Relation to ventricular and myocyte function, Circ Res 82 (1998) 482-495.

[25] F.G. Spinale, M. Tomita, J.L. Zellner, J.C. Cook, F.A. Crawford, M.R. Zile, Collagen remodeling and changes in Iv function during development and recovery from supraventricular tachycardia, Am J Physiol 261 (1991) H308-318.

[26] F.G. Spinale, J.L. Zellner, W.S. Johnson, D.M. Eble, P.D. Munyer, Cellular and extracellular remodeling with the development and recovery from tachycardia-induced cardiomyopathy: Changes in fibrillar collagen, myocyte adhesion capacity and proteoglycans, J Mol Cell Cardiol 28 (1996) 1591-1608.

[27] A.L. Chancey, G.L. Brower, J.T. Peterson, J.S. Janicki, Effects of matrix metalloproteinase inhibition on ventricular remodeling due to volume overload, Circulation 105 (2002) 1983-1988.

[28] J.T. Peterson, H. Hallak, L. Johnson, et al., Matrix metalloproteinase inhibition attenuates left ventricular remodeling and dysfunction in a rat model of progressive heart failure, Circulation 103 (2001) 2303-2309.

[29] L.E. Rohde, A. Ducharme, L.H. Arroyo, et al., Matrix metalloproteinase inhibition attenuates early left ventricular enlargement after experimental myocardial infarction in mice, Circulation 99 (1999) 3063-3070.

[30] P.W. Fedak, D.S. Smookler, Z. Kassiri, et al., Timp-3 deficiency leads to dilated cardiomyopathy, Circulation 110 (2004) 2401-2409.

[31] N. Nishikawa, K. Yamamoto, Y. Sakata, et al., Differential activation of matrix metalloproteinases in heart failure with and without ventricular dilatation, Cardiovasc Res 57 (2003) 766-774.

[32] N. Sivasubramanian, M.L. Coker, K.M. Kurrelmeyer, et al., Left ventricular remodeling in transgenic mice with cardiac restricted overexpression of tumor necrosis factor, Circulation 104 (2001) 826-831.

[33] J. Li, P.L. Schwimmbeck, C. Tschope, et al., Collagen degradation in a murine myocarditis model: Relevance of matrix metalloproteinase in association with inflammatory induction, Cardiovasc Res 56 (2002) 235-247.

[34] C. Cheung, H. Luo, B. Yanagawa, et al., Matrix metalloproteinases and tissue inhibitors of metalloproteinases in coxsackievirus-induced myocarditis, Cardiovasc Pathol 15 (2006) 63-74.

[35] S. Heymans, M. Pauschinger, A. De Palma, et al., Inhibition of urokinase-type plasminogen activator or matrix metalloproteinases prevents cardiac injury and dysfunction during viral myocarditis, Circulation 114 (2006) 565-573.

[36] Z. Szabo, J. Magga, T. Alakoski, et al., Connective tissue growth factor inhibition attenuates left ventricular remodeling and dysfunction in pressure overload-induced heart failure, Hypertension 63 (2014) 1235-1240.

[37] K.R. Hutchinson, A. Guggilam, M.J. Cismowski, et al., Temporal pattern of left ventricular structural and functional remodeling following reversal of volume overload heart failure, J Appl Physiol (1985) 111 (2011) 1778-1788. 
[38] Z. Gunja-Smith, A.R. Morales, R. Romanelli, J.F. Woessner, Jr., Remodeling of human myocardial collagen in idiopathic dilated cardiomyopathy. Role of metalloproteinases and pyridinoline cross-links, Am J Pathol 148 (1996) 1639-1648.

[39] W.G. Stetler-Stevenson, Dynamics of matrix turnover during pathologic remodeling of the extracellular matrix, Am J Pathol 148 (1996) 1345-1350.

[40] J.E. Bishop, R. Greenbaum, D.G. Gibson, M. Yacoub, G.J. Laurent, Enhanced deposition of predominantly type i collagen in myocardial disease, J Mol Cell Cardiol 22 (1990) 1157-1165.

[41] S. Khan, J. Joyce, K.B. Margulies, T. Tsuda, Enhanced bioactive myocardial transforming growth factor-beta in advanced human heart failure, Circ J 78 (2014) 2711-2718.

[42] S. Klotz, R.F. Foronjy, M.L. Dickstein, et al., Mechanical unloading during left ventricular assist device support increases left ventricular collagen cross-linking and myocardial stiffness, Circulation 112 (2005) 364374.

[43] M.M. Marijianowski, P. Teeling, J. Mann, A.E. Becker, Dilated cardiomyopathy is associated with an increase in the type i/type iii collagen ratio: A quantitative assessment, J Am Coll Cardiol 25 (1995) 1263-1272.

[44] M. Pauschinger, A. Doerner, A. Remppis, R. Tannhauser, U. Kuhl, H.P. Schultheiss, Differential myocardial abundance of collagen type $\mathrm{i}$ and type iii mrna in dilated cardiomyopathy: Effects of myocardial inflammation, Cardiovasc Res 37 (1998) 123-129.

[45] M. Pauschinger, D. Knopf, S. Petschauer, et al., Dilated cardiomyopathy is associated with significant changes in collagen type i/iii ratio, Circulation 99 (1999) 2750-2756.

[46] P. Sivakumar, S. Gupta, S. Sarkar, S. Sen, Upregulation of lysyl oxidase and mmps during cardiac remodeling in human dilated cardiomyopathy, Mol Cell Biochem 307 (2008) 159-167.

[47] H. Yoshikane, M. Honda, Y. Goto, S. Morioka, A. Ooshima, K. Moriyama, Collagen in dilated cardiomyopathy-scanning electron microscopic and immunohistochemical observations, Jpn Circ J 56 (1992) 899-910.

[48] H. Mollnau, B. Munkel, J. Schaper, Collagen vi in the extracellular matrix of normal and failing human myocardium, Herz 20 (1995) 89-94.

[49] H.K. Reddy, I.E. Tjahja, S.E. Campbell, J.S. Janicki, M.R. Hayden, S.C. Tyagi, Expression of matrix metalloproteinase activity in idiopathic dilated cardiomyopathy: A marker of cardiac dilatation, Mol Cell Biochem 264 (2004) 183-191.

[50] S.C. Tyagi, S.E. Campbell, H.K. Reddy, E. Tjahja, D.J. Voelker, Matrix metalloproteinase activity expression in infarcted, noninfarcted and dilated cardiomyopathic human hearts, Mol Cell Biochem 155 (1996) 13-21.

[51] S.C. Tyagi, S. Kumar, D.J. Voelker, H.K. Reddy, J.S. Janicki, J.J. Curtis, Differential gene expression of extracellular matrix components in dilated cardiomyopathy, J Cell Biochem 63 (1996) 185-198.

[52] P. Rouet-Benzineb, J.M. Buhler, P. Dreyfus, et al., Altered balance between matrix gelatinases (mmp-2 and mmp-9) and their tissue inhibitors in human dilated cardiomyopathy: Potential role of mmp-9 in myosinheavy chain degradation, Eur J Heart Fail 1 (1999) 337-352.

[53] F.G. Spinale, M.L. Coker, L.J. Heung, et al., A matrix metalloproteinase induction/activation system exists in the human left ventricular myocardium and is upregulated in heart failure, Circulation 102 (2000) 19441949.

[54] C.V. Thomas, M.L. Coker, J.L. Zellner, J.R. Handy, A.J. Crumbley, 3rd, F.G. Spinale, Increased matrix metalloproteinase activity and selective upregulation in Iv myocardium from patients with end-stage dilated cardiomyopathy, Circulation 97 (1998) 1708-1715. 
[55] G. Klappacher, P. Franzen, D. Haab, et al., Measuring extracellular matrix turnover in the serum of patients with idiopathic or ischemic dilated cardiomyopathy and impact on diagnosis and prognosis, Am J Cardiol 75 (1995) 913-918.

[56] B. Schwartzkopff, M. Fassbach, B. Pelzer, M. Brehm, B.E. Strauer, Elevated serum markers of collagen degradation in patients with mild to moderate dilated cardiomyopathy, Eur J Heart Fail 4 (2002) 439-434.

[57] A.T. Yan, R.T. Yan, F.G. Spinale, et al., Plasma matrix metalloproteinase-9 level is correlated with left ventricular volumes and ejection fraction in patients with heart failure, J Card Fail 12 (2006) 514-519.

[58] A. Jordan, V. Roldan, M. Garcia, et al., Matrix metalloproteinase-1 and its inhibitor, timp-1, in systolic heart failure: Relation to functional data and prognosis, J Intern Med 262 (2007) 385-392.

[59] Y. Naito, T. Tsujino, M. Lee-Kawabata, et al., Matrix metalloproteinase-1 and -2 levels are differently regulated in acute exacerbation of heart failure in patients with and without left ventricular systolic dysfunction, Heart Vessels 24 (2009) 181-186.

[60] F. Terasaki, H. Okamoto, K. Onishi, et al., Higher serum tenascin-c levels reflect the severity of heart failure, left ventricular dysfunction and remodeling in patients with dilated cardiomyopathy, Circ J 71 (2007) 327330.

[61] A. Tamura, S. Kusachi, K. Nogami, et al., Tenascin expression in endomyocardial biopsy specimens in patients with dilated cardiomyopathy: Distribution along margin of fibrotic lesions, Heart 75 (1996) 291-294.

[62] E. Herpel, S. Singer, C. Flechtenmacher, et al., Extracellular matrix proteins and matrix metalloproteinases differ between various right and left ventricular sites in end-stage cardiomyopathies, Virchows Arch 446 (2005) 369-378.

[63] K. Nogami, S. Kusachi, H. Nunoyama, et al., Extracellular matrix components in dilated cardiomyopathy. Immunohistochemical study of endomyocardial biopsy specimens, Jpn Heart J 37 (1996) 483-494.

[64] T.Y. Hsia, J.M. Ringewald, R.E. Stroud, et al., Determinants of extracellular matrix remodelling are differentially expressed in paediatric and adult dilated cardiomyopathy, Eur J Heart Fail 13 (2011) 271-277.

[65] A. Naba, K.R. Clauser, S. Hoersch, H. Liu, S.A. Carr, R.O. Hynes, The matrisome: In silico definition and in vivo characterization by proteomics of normal and tumor extracellular matrices, Mol Cell Proteomics 11 (2012) 9.

[66] A. Naba, K.R. Clauser, H. Ding, C.A. Whittaker, S.A. Carr, R.O. Hynes, The extracellular matrix: Tools and insights for the "omics" era, Matrix Biol 8 (2015) 00121-00123.

[67] T. Clausen, M. Kaiser, R. Huber, M. Ehrmann, Htra proteases: Regulated proteolysis in protein quality control, Nat Rev Mol Cell Biol 12 (2011) 152-162.

[68] I. Yana, S.J. Weiss, Regulation of membrane type-1 matrix metalloproteinase activation by proprotein convertases, Mol Biol Cell 11 (2000) 2387-2401.

[69] T. Pihlajaniemi, R. Myllyla, K.I. Kivirikko, Prolyl 4-hydroxylase and its role in collagen synthesis, J Hepatol 13 (1991) S2-7.

[70] H.L. Bader, L.W. Wang, J.C. Ho, et al., A disintegrin-like and metalloprotease domain containing thrombospondin type 1 motif-like 5 (adamtsl5) is a novel fibrillin-1-, fibrillin-2-, and heparin-binding member of the adamts superfamily containing a netrin-like module, Matrix Biol 31 (2012) 398-411.

[71] K. Tsutsui, R. Manabe, T. Yamada, et al., Adamtsl-6 is a novel extracellular matrix protein that binds to fibrillin-1 and promotes fibrillin-1 fibril formation, J Biol Chem 285 (2010) 4870-4882.

[72] F. Bost, M. Diarra-Mehrpour, J.P. Martin, Inter-alpha-trypsin inhibitor proteoglycan family--a group of proteins binding and stabilizing the extracellular matrix, Eur J Biochem 252 (1998) 339-346. 
[73] D. Bergqvist, I.M. Nilsson, Hereditary alpha 2-macroglobulin deficiency, Scand J Haematol 23 (1979) 433436.

[74] R.P. Somerville, J.M. Longpre, K.A. Jungers, et al., Characterization of adamts-9 and adamts-20 as a distinct adamts subfamily related to caenorhabditis elegans gon-1, J Biol Chem 278 (2003) 9503-9513.

[75] A.A. Bizet, K. Liu, N. Tran-Khanh, et al., The tgf-beta co-receptor, cd109, promotes internalization and degradation of tgf-beta receptors, Biochim Biophys Acta 5 (2011) 742-753.

[76] A. Bonnefoy, C. Legrand, Proteolysis of subendothelial adhesive glycoproteins (fibronectin, thrombospondin, and von willebrand factor) by plasmin, leukocyte cathepsin g, and elastase, Thromb Res 98 (2000) 323-332.

[77] P. Carmeliet, L. Moons, R. Lijnen, et al., Urokinase-generated plasmin activates matrix metalloproteinases during aneurysm formation, Nat Genet 17 (1997) 439-444.

[78] A. Leask, Tgfbeta, cardiac fibroblasts, and the fibrotic response, Cardiovasc Res 74 (2007) 207-212.

[79] D. Gospodarowicz, Localisation of a fibroblast growth factor and its effect alone and with hydrocortisone on 3 t3 cell growth, Nature 249 (1974) 123-127.

[80] S. Bouleau, H. Grimal, V. Rincheval, et al., Fgf1 inhibits p53-dependent apoptosis and cell cycle arrest via an intracrine pathway, Oncogene 24 (2005) 7839-7849.

[81] C.J. Powers, S.W. McLeskey, A. Wellstein, Fibroblast growth factors, their receptors and signaling, Endocr Relat Cancer 7 (2000) 165-197.

[82] Y.S. Li, P.G. Milner, A.K. Chauhan, et al., Cloning and expression of a developmentally regulated protein that induces mitogenic and neurite outgrowth activity, Science 250 (1990) 1690-1694.

[83] E.T. Bowden, G.E. Stoica, A. Wellstein, Anti-apoptotic signaling of pleiotrophin through its receptor, anaplastic lymphoma kinase, J Biol Chem 277 (2002) 35862-35868.

[84] K.D. Panse, L.E. Felkin, M.M. Lopez-Olaneta, et al., Follistatin-like 3 mediates paracrine fibroblast activation by cardiomyocytes, J Cardiovasc Transl Res 5 (2012) 814-826.

[85] G.C. Elson, P. Graber, C. Losberger, et al., Cytokine-like factor-1, a novel soluble protein, shares homology with members of the cytokine type i receptor family, J Immunol 161 (1998) 1371-1379.

[86] D. Sciaky, W. Brazer, D.M. Center, W.W. Cruikshank, T.J. Smith, Cultured human fibroblasts express constitutive il-16 mrna: Cytokine induction of active il-16 protein synthesis through a caspase-3-dependent mechanism, J Immunol 164 (2000) 3806-3814.

[87] X. Liu, A.M. Das, J. Seideman, et al., The cc chemokine ligand 2 ( $\mathrm{ccl} 2$ ) mediates fibroblast survival through il-6, Am J Respir Cell Mol Biol 37 (2007) 121-128.

[88] M. Orlandini, L. Marconcini, R. Ferruzzi, S. Oliviero, Identification of a c-fos-induced gene that is related to the platelet-derived growth factor/vascular endothelial growth factor family, Proc Natl Acad Sci U S A 93 (1996) 11675-11680.

[89] I. Puxeddu, R. Bader, A.M. Piliponsky, R. Reich, F. Levi-Schaffer, N. Berkman, The cc chemokine eotaxin/ccl11 has a selective profibrogenic effect on human lung fibroblasts, J Allergy Clin Immunol 117 (2006) 103-110.

[90] A. Iwabu, K. Smith, F.D. Allen, D.A. Lauffenburger, A. Wells, Epidermal growth factor induces fibroblast contractility and motility via a protein kinase c delta-dependent pathway, J Biol Chem 279 (2004) 1455114560.

[91] K.J. Lembach, Induction of human fibroblast proliferation by epidermal growth factor (egf): Enhancement by an egf-binding arginine esterase and by ascorbate, Proc Natl Acad Sci U S A 73 (1976) 183-187.

[92] B. Westermark, E. Blomquist, Stimulation of fibroblast migration by epidermal growth factor, Cell Biol Int Rep 4 (1980) 649-654. 
[93] D.H. You, M.J. Nam, Effects of human epidermal growth factor gene-transfected mesenchymal stem cells on fibroblast migration and proliferation, Cell Prolif 46 (2013) 408-415.

[94] R. Kajanne, P. Miettinen, A. Mehlem, et al., Egf-r regulates mmp function in fibroblasts through mapk and ap-1 pathways, J Cell Physiol 212 (2007) 489-497.

[95] K. Yamane, Y. Asano, K. Tamaki, H. Ihn, Epidermal growth factor up-regulates transforming growth factorbeta receptor type ii in human dermal fibroblasts via p38 mitogen-activated protein kinase pathway, Biochem Biophys Res Commun 352 (2007) 69-77.

[96] E.C. LeRoy, M.I. Trojanowska, E.A. Smith, Cytokines and human fibrosis, Eur Cytokine Netw 1 (1990) 215219.

[97] S. Struyf, P. Proost, J. Vandercappellen, et al., Synergistic up-regulation of mcp-2/ccl8 activity is counteracted by chemokine cleavage, limiting its inflammatory and anti-tumoral effects, Eur J Immunol 39 (2009) 843-857.

[98] J. Heino, The collagen family members as cell adhesion proteins, Bioessays 29 (2007) 1001-1010.

[99] R.J. Wenstrup, J.B. Florer, E.W. Brunskill, S.M. Bell, I. Chervoneva, D.E. Birk, Type v collagen controls the initiation of collagen fibril assembly, J Biol Chem 279 (2004) 53331-53337.

[100] G.R. Monroe, M. Harakalova, S.N. van der Crabben, et al., Familial ehlers-danlos syndrome with lethal arterial events caused by a mutation in col5a1, Am J Med Genet A 167 (2015) 1196-1203.

[101] M. van der Rest, R. Garrone, Collagen family of proteins, FASEB J 5 (1991) 2814-2823.

[102] C. Walchli, M. Koch, M. Chiquet, B.F. Odermatt, B. Trueb, Tissue-specific expression of the fibril-associated collagens xii and xiv, J Cell Sci 107 ( Pt 2) (1994) 669-681.

[103] J. Fitzgerald, J.F. Bateman, A new facit of the collagen family: Col21a1, FEBS Lett 505 (2001) 275-280.

[104] G. Tao, A.K. Levay, J.D. Peacock, et al., Collagen xiv is important for growth and structural integrity of the myocardium, J Mol Cell Cardiol 53 (2012) 626-638.

[105] T. Van Agtmael, M.A. Bailey, U. Schlotzer-Schrehardt, et al., Col4a1 mutation in mice causes defects in vascular function and low blood pressure associated with reduced red blood cell volume, Hum Mol Genet 19 (2010) 1119-1128.

[106] D.B. Gould, F.C. Phalan, S.E. van Mil, et al., Role of col4a1 in small-vessel disease and hemorrhagic stroke, N Engl J Med 354 (2006) 1489-1496.

[107] E. Verbeek, M.E. Meuwissen, F.W. Verheijen, et al., Col4a2 mutation associated with familial porencephaly and small-vessel disease, Eur J Hum Genet 20 (2012) 844-851.

[108] E. Adiguzel, G. Hou, D. Mulholland, et al., Migration and growth are attenuated in vascular smooth muscle cells with type viii collagen-null alleles, Arterioscler Thromb Vasc Biol 26 (2006) 56-61.

[109] R. Pankov, K.M. Yamada, Fibronectin at a glance, J Cell Sci 115 (2002) 3861-3863.

[110] M. Hyytiainen, C. Penttinen, J. Keski-Oja, Latent tgf-beta binding proteins: Extracellular matrix association and roles in tgf-beta activation, Crit Rev Clin Lab Sci 41 (2004) 233-264.

[111] S.L. Dallas, P. Sivakumar, C.J. Jones, et al., Fibronectin regulates latent transforming growth factor-beta (tgf beta) by controlling matrix assembly of latent tgf beta-binding protein-1, J Biol Chem 280 (2005) 18871-18880.

[112] T. Kanzaki, R. Shiina, Y. Saito, H. Oohashi, N. Morisaki, Role of latent tgf-beta 1 binding protein in vascular remodeling, Biochem Biophys Res Commun 246 (1998) 26-30.

[113] L. Zilberberg, V. Todorovic, B. Dabovic, et al., Specificity of latent tgf-beta binding protein (Itbp) incorporation into matrix: Role of fibrillins and fibronectin, J Cell Physiol 227 (2012) 3828-3836. 
[114] M.H. Konstandin, H. Toko, G.M. Gastelum, et al., Fibronectin is essential for reparative cardiac progenitor cell response after myocardial infarction, Circ Res 113 (2013) 115-125.

[115] A. van Dijk, H.W. Niessen, W. Ursem, J.W. Twisk, F.C. Visser, F.J. van Milligen, Accumulation of fibronectin in the heart after myocardial infarction: A putative stimulator of adhesion and proliferation of adiposederived stem cells, Cell Tissue Res 332 (2008) 289-298.

[116] M.M. Chen, A. Lam, J.A. Abraham, G.F. Schreiner, A.H. Joly, Ctgf expression is induced by tgf- beta in cardiac fibroblasts and cardiac myocytes: A potential role in heart fibrosis, J Mol Cell Cardiol 32 (2000) 18051819.

[117] K. Frazier, S. Williams, D. Kothapalli, H. Klapper, G.R. Grotendorst, Stimulation of fibroblast cell growth, matrix production, and granulation tissue formation by connective tissue growth factor, J Invest Dermatol 107 (1996) 404-411.

[118] A. Kato, O. Okamoto, K. Ishikawa, et al., Dermatopontin interacts with fibronectin, promotes fibronectin fibril formation, and enhances cell adhesion, J Biol Chem 286 (2011) 14861-14869.

[119] X. Liu, L. Meng, Q. Shi, et al., Dermatopontin promotes adhesion, spreading and migration of cardiac fibroblasts in vitro, Matrix Biol 32 (2013) 23-31.

[120] K. Tanaka, T. Arao, M. Maegawa, et al., Srpx2 is overexpressed in gastric cancer and promotes cellular migration and adhesion, Int J Cancer 124 (2009) 1072-1080.

[121] K. Ohashi, T. Enomoto, Y. Joki, et al., Neuron-derived neurotrophic factor functions as a novel modulator that enhances endothelial cell function and revascularization processes, J Biol Chem 289 (2014) 1413214144.

[122] P. Bornstein, S. Devarayalu, P. Li, C.M. Disteche, P. Framson, A second thrombospondin gene in the mouse is similar in organization to thrombospondin 1 but does not respond to serum, Proc Natl Acad Sci U S A 88 (1991) 8636-8640.

[123] J.E. Murphy-Ullrich, S. Gurusiddappa, W.A. Frazier, M. Hook, Heparin-binding peptides from thrombospondins 1 and 2 contain focal adhesion-labilizing activity, J Biol Chem 268 (1993) 26784-26789.

[124] Z. Yang, T.R. Kyriakides, P. Bornstein, Matricellular proteins as modulators of cell-matrix interactions: Adhesive defect in thrombospondin 2-null fibroblasts is a consequence of increased levels of matrix metalloproteinase-2, Mol Biol Cell 11 (2000) 3353-3364.

[125] J. Beattie, M. Kreiner, G.J. Allan, D.J. Flint, D. Domingues, C.F. van der Walle, Igfbp-3 and igfbp-5 associate with the cell binding domain (cbd) of fibronectin, Biochem Biophys Res Commun 381 (2009) 572-576.

[126] H. Zeng, Y. Zhang, Q. Yi, Y. Wu, R. Wan, L. Tang, Crim1, a newfound cancer-related player, regulates the adhesion and migration of lung cancer cells, Growth Factors 33 (2015) 384-392. 


\section{Tables and figures}

Table 1. Summary of ECM response in myocardial fibrosis associated with DCM and SHF in animal studies

\section{Name \\ Core matrisome \\ Glycoprotein}

Expression

Study model

References

ELN Elastin

mRNA $\uparrow$

ACF-induced rat

Hutchinson et al. 2011

\section{Collagen}

COL1 Collagen, type I

mRNA $\uparrow \quad$ SHF rat

Nishikawa et al. 2003

ACF-induced rat

Hutchinson et al. 2011

Protein $\uparrow \quad$ SHF rat

Woodiwiss et al. 2001

COL3 Collagen, type III

mRNA $\uparrow \quad$ ACF-induced rat

Hutchinson et al. 2011

Protein $\uparrow$

BIO 53.58 hamster

Okada et al. 1996

\section{Matrisome-associated}

ECM-affiliated

GSPG

Chondroitin sulfate

proteoglycan

Protein $\uparrow$

Pacing-induced CM

Spinale et al. 1996

pig

\section{ECM regulator}

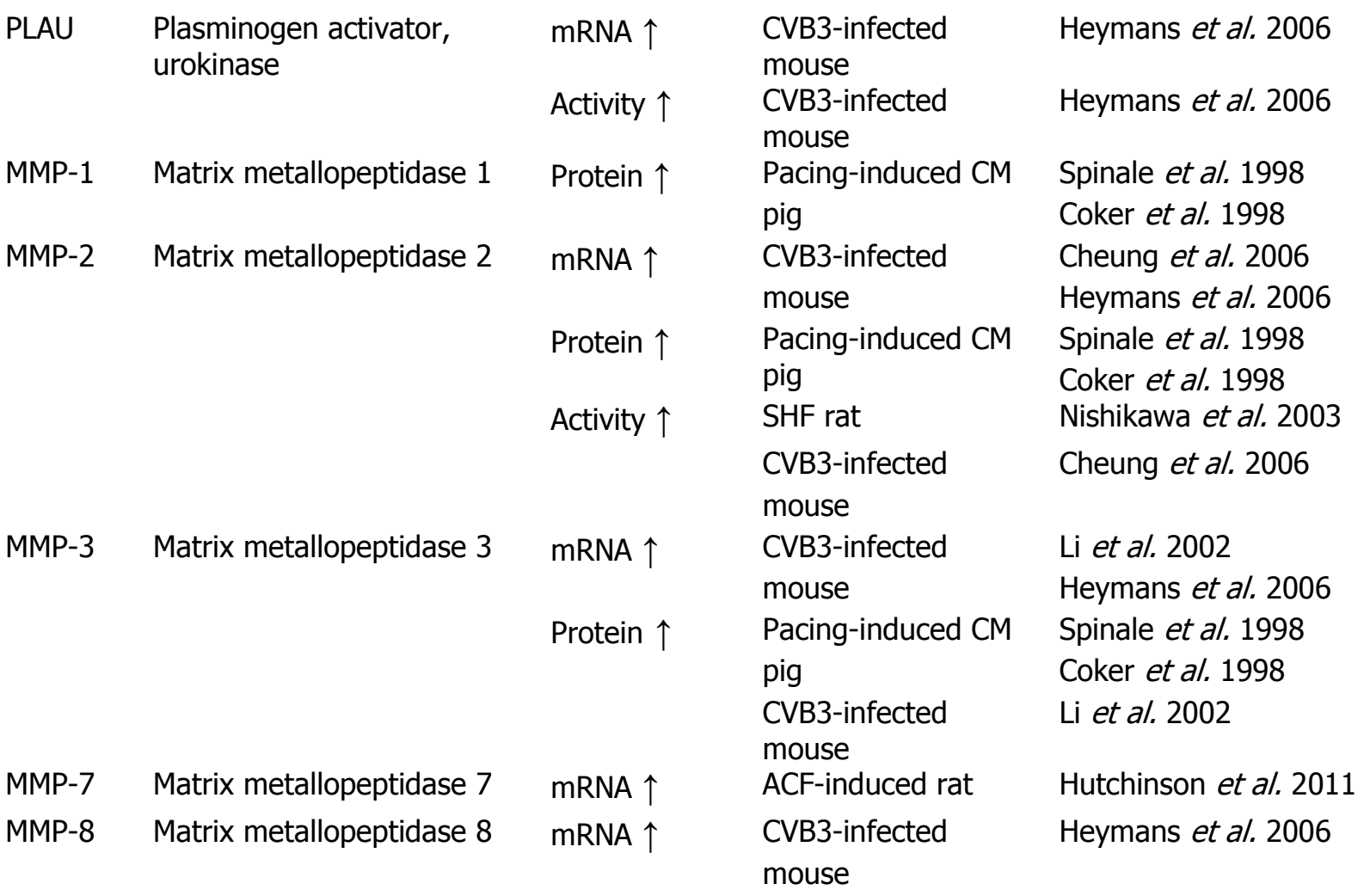




\begin{tabular}{|c|c|c|c|c|}
\hline \multirow[t]{6}{*}{ MMP-9 } & Matrix metallopeptidase 9 & mRNA $\uparrow$ & $\begin{array}{l}\text { CVB3-infected } \\
\text { mouse }\end{array}$ & $\begin{array}{l}\text { Li et al. } 2002 \\
\text { Cheung et al. } 2006\end{array}$ \\
\hline & & Protein $\uparrow$ & CVB3-infected & $\begin{array}{l}\text { Heymans et al. } 2006 \\
\text { Li et al. } 2002\end{array}$ \\
\hline & & & mouse & Heymans et al. 2006 \\
\hline & & Activity $\uparrow$ & SHF rat & Nishikawa et al. 2003 \\
\hline & & & CVB3-infected & Cheung et al. 2006 \\
\hline & & & mouse & Heymans et al. 2006 \\
\hline MMP-12 & Matrix metallopeptidase 12 & mRNA $\uparrow$ & $\begin{array}{l}\text { CVB3-infected } \\
\text { mouse }\end{array}$ & Cheung et al. 2006 \\
\hline MMP-13 & Matrix metallopeptidase 13 & mRNA $\uparrow$ & $\begin{array}{l}\text { CVB3-infected } \\
\text { mouse }\end{array}$ & Heymans et al. 2006 \\
\hline MMP-14 & Matrix metallopeptidase 14 & mRNA $\downarrow$ & $\begin{array}{l}\text { CVB3-infected } \\
\text { mouse }\end{array}$ & Li et al. 2002 \\
\hline \multirow[t]{2}{*}{ TIMP-1 } & Tissue inhibitor of & mRNA $\uparrow$ & SHF rat & Nishikawa et al. 2003 \\
\hline & & mRNA $\downarrow$ & $\begin{array}{l}\text { CVB3-infected } \\
\text { mouse }\end{array}$ & Li et al. 2002 \\
\hline \multirow[t]{2}{*}{ TIMP-2 } & $\begin{array}{l}\text { Tissue inhibitor of } \\
\text { metalloproteinases } 2\end{array}$ & mRNA $\uparrow$ & SHF rat & Nishikawa et al. 2003 \\
\hline & & mRNA $\uparrow$ & ACF-induced rat & Hutchinson et al. 2011 \\
\hline TIMP-3 & $\begin{array}{l}\text { Tissue inhibitor of } \\
\text { metalloproteinases } 3\end{array}$ & Protein $\downarrow$ & $\begin{array}{l}\text { CVB3-infected } \\
\text { mouse }\end{array}$ & Cheung et al. 2006 \\
\hline TIMP-4 & $\begin{array}{l}\text { Tissue inhibitor of } \\
\text { metalloproteinases } 4\end{array}$ & Protein $\downarrow$ & $\begin{array}{l}\text { CVB3-infected } \\
\text { mouse }\end{array}$ & $\begin{array}{l}\text { Li et al. } 2002 \\
\text { Cheung et al. } 2006\end{array}$ \\
\hline
\end{tabular}


Table 2. Summary of ECM response in myocardial fibrosis associated with DCM and SHF in human studies

\begin{tabular}{|ll}
\hline Name & Function \\
Core & matrisome \\
Glycoprotein \\
LAM & Laminin \\
TNC & Tenascin C
\end{tabular}

\section{Collagen}

COL1 Collagen, type I

COL3 Collagen, type III

\section{Matrisome-associated \\ ECM regulator}

LOX Lysyl oxidase

MMP-1 Matrix metallopeptidase 1

MMP-2 Matrix metallopeptidase 2

MMP-7 Matrix metallopeptidase 7

MMP-8 Matrix metallopeptidase 8

MMP-9 Matrix metallopeptidase 9

MMP-14 Matrix metallopeptidase 14

TIMP-1 Tissue inhibitor of metalloproteinases 1
Expression Study model

\section{References}

$\begin{array}{llll}\text { Protein } \uparrow & \text { DCM } & \text { (serum) } & \text { Klappacher et al. } 1995 \\ \text { Protein } \uparrow & \text { DCM } & \text { (serum) } & \text { Terasaki et al. 2007 }\end{array}$

$\begin{array}{ll}\text { mRNA } \uparrow & \text { DCM } \\ \text { Protein } \uparrow & \text { DCM }\end{array}$

mRNA $\uparrow \quad$ DCM

Protein $\uparrow \quad$ DCM

Pauschinger et al. 1999

Khan et al. 2014

Bishop et al. 1990

Klotz et al. 2005

Sivakumar et al. 2008

Pauschinger et al. 1999

Khan et al. 2014

Bishop et al. 1990

Klotz et al. 2005

Sivakumar et al. 2008

$\begin{array}{ll}\text { mRNA } \uparrow & \text { DCM } \\ \text { Protein } \uparrow & \text { DCM } \\ \text { mRNA } \uparrow & \text { DCM } \\ \text { Protein } \uparrow & \text { SHF } \\ & \text { DCM }\end{array}$

Protein $\downarrow$

SHF

DCM

(serum) Swartzkopff et al. 2002

(serum) Jordán et al. 2007

Spinale et al. 2000

Thomas et al. 1998

Protein $\uparrow \quad$ SHF

(serum)

Yan et al. 2006

(serum) Naito et al. 2009

DCM

Sivakumar et al. 2008

Spinale et al. 2000

Protein $\uparrow \quad$ DCM

Protein $\downarrow \quad$ pDCM

Spinale et al. 2000

Thomas et al. 1998

Hsia et al. 2011

Protein $\downarrow \quad$ pDCM

Protein $\uparrow \quad$ pDCM

Protein $\uparrow \quad$ DCM

pDCM

Protein $\uparrow \quad$ DCM

mRNA $\uparrow \quad$ DCM

Hsia et al. 2011

Hsia et al. 2011

Klotz et al. 2005

Sivakumar et al. 2008

Spinale et al. 2000

Thomas et al. 1998

Hsia et al. 2011

Spinale et al. 2000

Sivakumar et al. 2008

Rouet-Benzineb et al. 


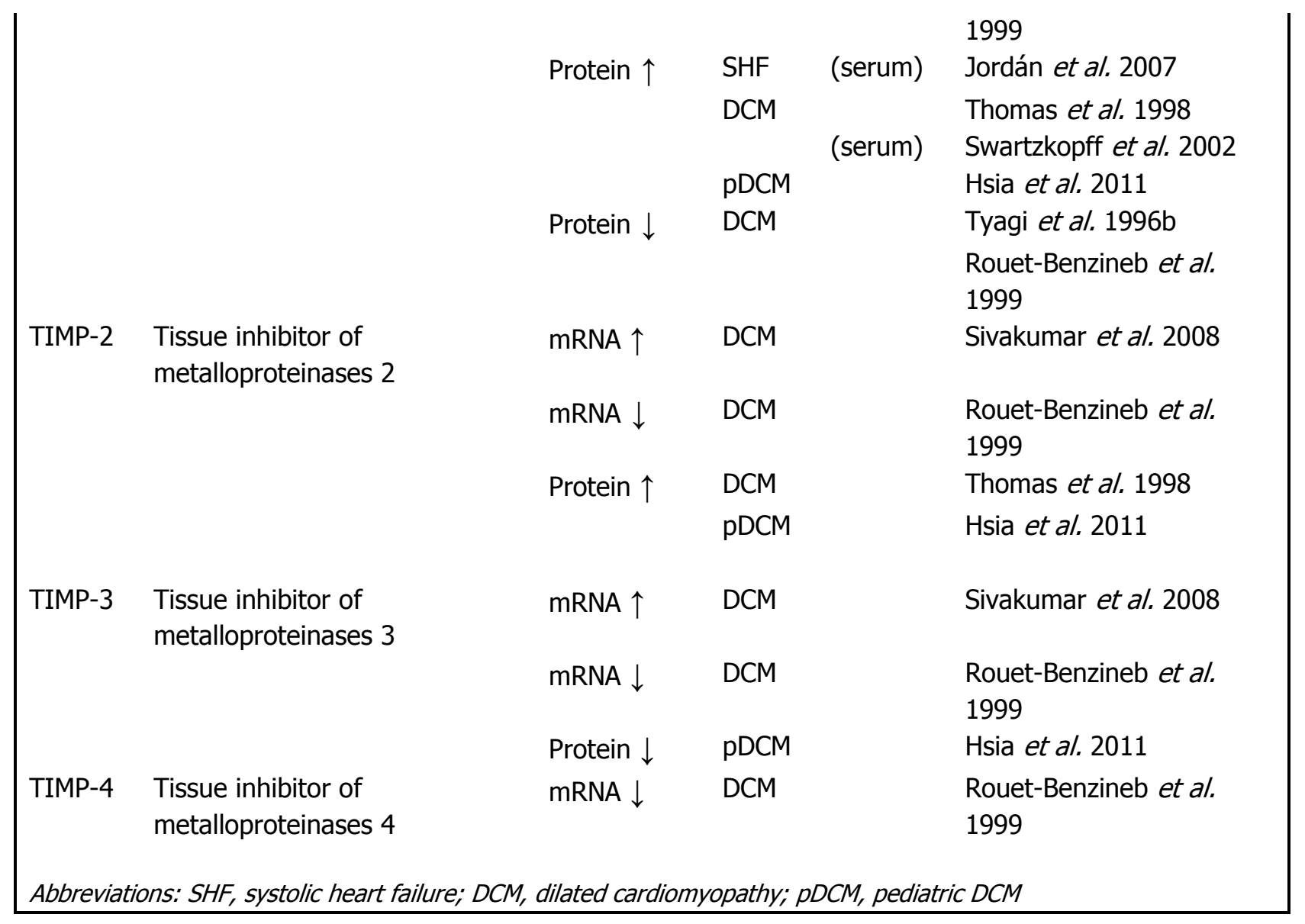


Table 3. Characteristics of the patient groups used in three dilated cardiomyopathy GEO datasets

\begin{tabular}{|clll|}
\hline & GSE42955 & GSE3586 & GSE3585 \\
\hline Tissue & $\begin{array}{l}\text { Left ventricular; } \\
\text { Transmural near the apex }\end{array}$ & Cardiac septum & $\begin{array}{l}\text { Left ventricular; } \\
\text { subendocardial }\end{array}$ \\
\hline Sample size & 17 & 28 & 12 \\
\hline DCM & 12 & 13 & 7 \\
\hline Control & 5 & 15 & 5 \\
\hline Gender & & & 7 \\
\hline DCM & 12 & 13 & 4 \\
\hline Male & 12 & 8 & 3 \\
\hline Female & 0 & 5 & 5 \\
\hline Control & 5 & 15 & 0 \\
\hline Male & 5 & 11 & 5 \\
\hline Female & 0 & 4 & Caucasian \\
\hline Ethnicity & N/A & Caucasian & $37 \pm 14$ \\
\hline Age (yrs.) & & & $49 \pm 9$ \\
\hline DCM & $48 \pm 9$ & $44 \pm 18$ & $25 \pm 6$ \\
\hline Control & N/A & $54 \pm 4$ & \\
\hline EF DCM (\%) & $20 \pm 6$ & $21 \pm 9$ & \\
\hline
\end{tabular}


Table 4. Number of genes identified as upregulated in three dilated cardiomyopathy GEO datasets and further classified in matrisome subgroups

\begin{tabular}{|clll|}
\hline & GSE42955 & GSE3586 & GSE3585 \\
\hline Total no. of genes & 972 & 3877 & 927 \\
\hline Matrisome & $49(5 \%)$ & $154(4 \%)$ & $84(9 \%)$ \\
\hline Non-matrisome & $923(95 \%)$ & $3723(96 \%)$ & $843(91 \%)$ \\
\hline Core matrisome & & $52(62 \%)$ \\
\hline Glycoproteins & $24(49 \%)$ & $60(39 \%)$ & $32(62 \%)$ \\
\hline Collagens & $15(62 \%)$ & $38(63 \%)$ & $12(23 \%)$ \\
\hline Proteoglycans & $6(25 \%)$ & $12(20 \%)$ & $8(15 \%)$ \\
\hline & $3(13 \%)$ & $10(17 \%)$ & $32(38 \%)$ \\
\hline Matrisome associated & & & $6(19 \%)$ \\
\hline ECM-affiliated genes & $25(51 \%)$ & $94(61 \%)$ & $14(44 \%)$ \\
\hline ECM regulators & $4(16 \%)$ & $25(27 \%)$ & $12(37 \%)$ \\
\hline Secreted factors & $8(32 \%)$ & $30(32 \%)$ & $0(0 \%)$ \\
\hline Retired & $13(52 \%)$ & $39(41 \%)$ & \\
\hline
\end{tabular}

\begin{tabular}{|c|c|c|c|}
\hline & GSE42955 & GSE3586 & GSE3585 \\
\hline Total no. of genes & 1223 & 3508 & 546 \\
\hline Matrisome & $49(4 \%)$ & $160(5 \%)$ & $31(6 \%)$ \\
\hline Non-matrisome & $1174(96 \%)$ & $3348(95 \%)$ & $515(94 \%)$ \\
\hline Core matrisome & $4(8 \%)$ & $50(31 \%)$ & $5(16 \%)$ \\
\hline Glycoproteins & $4(100 \%)$ & $38(76 \%)$ & $2(40 \%)$ \\
\hline Collagens & $0(0 \%)$ & $8(16 \%)$ & $1(20 \%)$ \\
\hline Proteoglycans & $0(0 \%)$ & $4(8 \%)$ & $2(40 \%)$ \\
\hline Matrisome associated & $45(92 \%)$ & $109(68 \%)$ & $26(84 \%)$ \\
\hline ECM-affiliated genes & $10(22 \%)$ & $20(18 \%)$ & $5(19 \%)$ \\
\hline ECM regulators & $11(25 \%)$ & $43(40 \%)$ & $8(31 \%)$ \\
\hline Secreted factors & $24(53 \%)$ & $46(42 \%)$ & $13(50 \%)$ \\
\hline Retired & $0(0 \%)$ & $1(1 \%)$ & $0(0 \%)$ \\
\hline
\end{tabular}




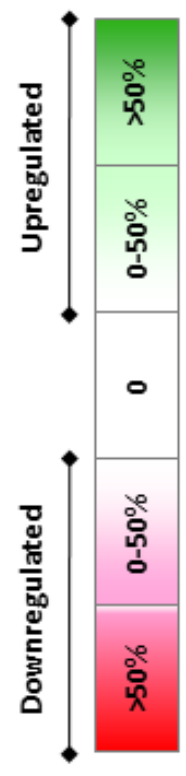

\begin{tabular}{|c|c|c|c|c|c|c|c|}
\hline & & & & logFC GSE42955 & logFC GSE3586 I & logFC GSE3585 & P-value \\
\hline & & & ADAMTSL5 & 0.38 & 0.28 & 0.00 & $1.93 E-03$ \\
\hline & & & & 0.00 & 0.14 & 0.22 & $3.21 \mathrm{E}-02$ \\
\hline & & & EGLN3 & 0.64 & 0.15 & 0.55 & $1.71 E-02$ \\
\hline & & & HTRA1 & 0.00 & 0.95 & 0.62 & $5.25 E-04$ \\
\hline & 兄 & & ITIH5 & 0.54 & 0.25 & 0.00 & $2.43 E-03$ \\
\hline & 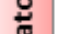 & & P4HA2 & 0.45 & 0.11 & 0.43 & $1.37 E-02$ \\
\hline & $\frac{\pi}{3}$ & & PCSK5 & 0.45 & 0.00 & 0.45 & $8.94 E-03$ \\
\hline & & & PCSK6 & 0.00 & 0.17 & 0.43 & $2.63 E-02$ \\
\hline & $\ddot{x}$ & & PLOD1 & 0.39 & 0.00 & 0.20 & $1.45 E-02$ \\
\hline & 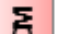 & & A2M & -0.46 & -0.21 & -0.31 & $8.79 E-03$ \\
\hline & & & ADAMTS9 & 0.00 & -0.31 & -0.53 & $5.71 E-04$ \\
\hline & & & CD109 & -0.70 & -0.12 & 0.00 & $2.34 E-03$ \\
\hline & & & F13A1 & 0.00 & -0.65 & -0.92 & $7.77 E-03$ \\
\hline & & & PLAU & -0.28 & 0.00 & -0.63 & $2.508-02$ \\
\hline हु & & & SERPINA3 & -1.84 & 0.00 & -0.81 & $3.51 E-03$ \\
\hline & & & ANXA7 & 0.00 & 0.14 & 0.19 & $2.28 E-02$ \\
\hline 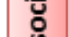 & छ & & SDC2 & 0.00 & 0.34 & 0.40 & $2.38 E-02$ \\
\hline & 荤 & & CD209 & 0.00 & -0.14 & -0.61 & $7.01 E-03$ \\
\hline & 点 & & COLEC10 & -0.43 & -0.31 & 0.00 & $1.32 E-03$ \\
\hline$\stackrel{\square}{E}$ & 安 & & FCN3 & -0.98 & 0.00 & -1.24 & $1.33 E-02$ \\
\hline & $\bar{f}$ & & SEMA3D & -0.41 & -0.26 & 0.00 & $2.898-03$ \\
\hline . & 总 & & SEMA4B & -0.53 & -0.15 & 0.00 & $8.57 E-03$ \\
\hline 带 & & & CRLF1 & 0.00 & 0.10 & 0.78 & $1.50 E-02$ \\
\hline & & & FGF1 & 0.00 & 0.51 & 0.43 & $1.25 E-02$ \\
\hline & & & FRZB & 0.95 & 0.00 & 1.64 & $2.40 E-03$ \\
\hline & & & FSTL3 & 0.71 & 0.00 & 0.61 & $8.968-04$ \\
\hline & 는 & & IL16 & 0.20 & 0.15 & 0.00 & $2.04 E-02$ \\
\hline & U & & PTN & 0.58 & 0.36 & 0.00 & $1.03 E-02$ \\
\hline & $\sqrt{2}$ & & SFRP4 & 0.90 & 0.00 & 1.27 & $5.97 E-03$ \\
\hline & $\bar{\nabla}$ & & CCL11 & 0.00 & -0.23 & -0.78 & $5.27 E-04$ \\
\hline & 迆 & & CCL2 & -1.86 & -1.08 & -1.46 & $9.05 E-04$ \\
\hline & 는 & & CCL8 & -0.77 & 0.00 & -0.68 & $3.50 E-02$ \\
\hline & J & & EGF & 0.00 & -0.12 & -0.26 & $2.81 E-02$ \\
\hline & & & FGF7 & -0.39 & 0.00 & -0.41 & $4.53 \mathrm{E}-02$ \\
\hline & & & FIGF & -1.01 & 0.00 & -0.68 & $3.61 E-02$ \\
\hline & & & IL6 & -0.67 & -0.14 & -0.26 & $2.59 E-02$ \\
\hline & & & S100A12 & -0.31 & 0.00 & -0.29 & $2.72 E-02$ \\
\hline & 5 & & ASPN & 0.00 & 0.88 & 1.31 & $2.73 E-04$ \\
\hline & 8 & & BGN & 0.00 & 0.23 & 0.73 & $2.50 E-02$ \\
\hline & $\overline{00}$ & & LUM & 0.64 & 0.81 & 0.00 & $1.74 E-02$ \\
\hline & ฮं & & OGN & 0.82 & 0.00 & 1.19 & $101 E-02$ \\
\hline & ธँ & & SPOCK1 & 0.00 & 0.34 & 1.35 & $1.098-02$ \\
\hline & 농 & & CTGF & 0.00 & 0.52 & 1.92 & $2.20 E-03$ \\
\hline & & & DPT & 0.60 & 0.00 & 0.74 & $2.75 \varepsilon-02$ \\
\hline & & & ECM2 & 0.64 & 0.28 & 0.79 & $1.34 E-03$ \\
\hline & & & ELN & 0.25 & 0.00 & 1.23 & $1.16 \varepsilon-02$ \\
\hline & & & FN1 & 0.00 & 0.12 & 0.73 & $1.97 E-02$ \\
\hline & & & IGFBP3 & 0.46 & 0.00 & 0.83 & $1.968-03$ \\
\hline & n & & LAMA2 & 0.39 & 0.00 & 0.55 & $1.68 \mathrm{E}-02$ \\
\hline 5 & 尌 & & LAMB1 & 0.37 & 0.40 & 0.65 & $2.33 E-03$ \\
\hline 5 & ธัँ & & LTBP1 & 0.00 & 0.27 & 0.80 & $3.53 E-03$ \\
\hline 요 & 늠 & & LTBP2 & 0.00 & 0.71 & 1.29 & $5.668-04$ \\
\hline$\Sigma$ & $\overline{8}$ & & NDNF & 0.51 & 0.24 & 0.00 & $1.53 E-02$ \\
\hline & $\supseteq$ & & PCOLCE2 & 0.00 & 0.58 & 0.91 & $1.01 E-03$ \\
\hline 3 & $\overline{5}$ & & SPON1 & 0.65 & 0.00 & 0.70 & $1.97 E-02$ \\
\hline & & & SRPX2 & 0.53 & 0.00 & 0.33 & $2.968-02$ \\
\hline & & & SVEP1 & 0.00 & 0.21 & 0.45 & $3.07 E-03$ \\
\hline & & & TSP2 & 0.00 & 0.14 & 1.03 & $1.55 \mathrm{E}-03$ \\
\hline & & & CRIM1 & -0.42 & -0.12 & 0.00 & $1.71 E-02$ \\
\hline & & & IGFBP4 & 0.00 & -0.20 & -0.50 & 1. $55 \mathrm{E}-02$ \\
\hline & & & MMRN2 & -0.40 & -0.15 & 0.00 & $3.73 E-04$ \\
\hline & & & COL14A1 & 0.00 & 0.14 & 0.90 & $1.35 E-02$ \\
\hline & & & COL1A1 & 0.51 & 0.00 & 1.15 & $3.098-02$ \\
\hline & & & COL1A2 & 0.49 & 0.00 & 1.16 & $2.45 E-02$ \\
\hline & 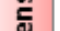 & & COL21A1 & 0.00 & 0.58 & 0.41 & $1.29 \mathrm{E}-02$ \\
\hline & 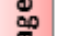 & & COL3A1 & 0.00 & 0.70 & 2.75 & $2.92 E-02$ \\
\hline & 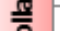 & & COLAA1 & 0.33 & 0.00 & 0.39 & $9.47 E-03$ \\
\hline & 8 & & COLAA2 & 0.22 & 0.00 & 0.29 & $3.22 E-02$ \\
\hline & & & COL5A1 & 0.00 & 0.33 & 0.64 & $2.03 E-03$ \\
\hline & & & COL5A2 & 0.45 & 0.00 & 0.78 & 8.11E-03 \\
\hline & & & COL8A1 & 0.37 & 0.32 & 0.35 & $8.41 E-03$ \\
\hline
\end{tabular}

Figure 1. Heatmap composed of genes found to be significantly up-or downregulated in dilated cardiomyopathy based on three GEO datasets. Only genes differentially expressed in two or more datasets where included. Expression levels are displayed in $\log ^{2}$ fold change. 


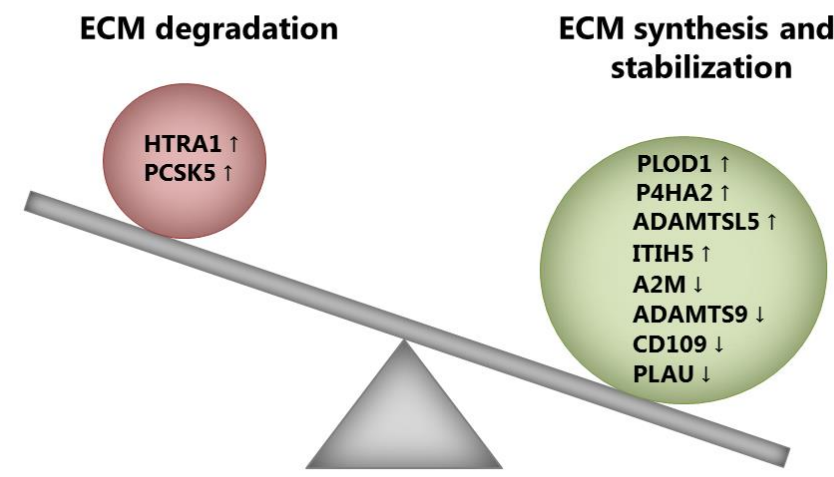

Figure 2. Hypothetical schematic representation of the involvement of ECM regulators in dilated cardiomyopathy.
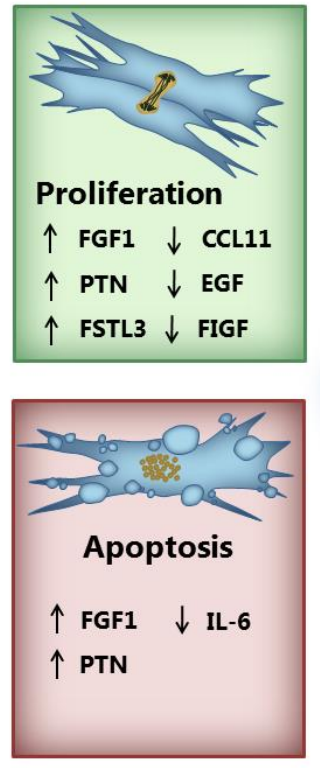

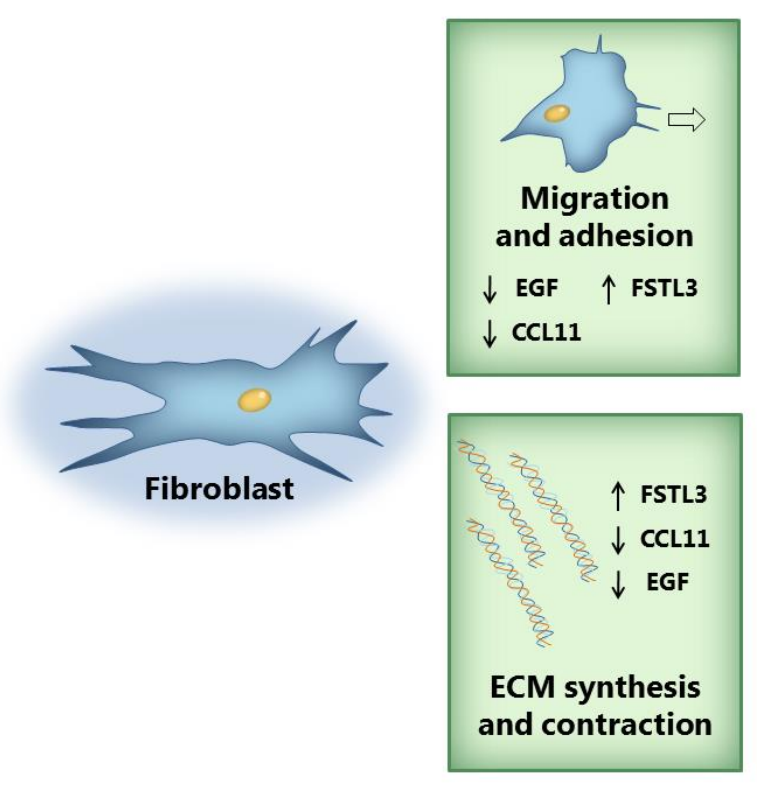

Figure 3. Hypothetical schematic representation of the effects of secreted factors within the extracellular matrix on fibroblast activity in dilated cardiomyopathy. Green framing represents stimulation of the process mentioned, red framing represents inhibition. 

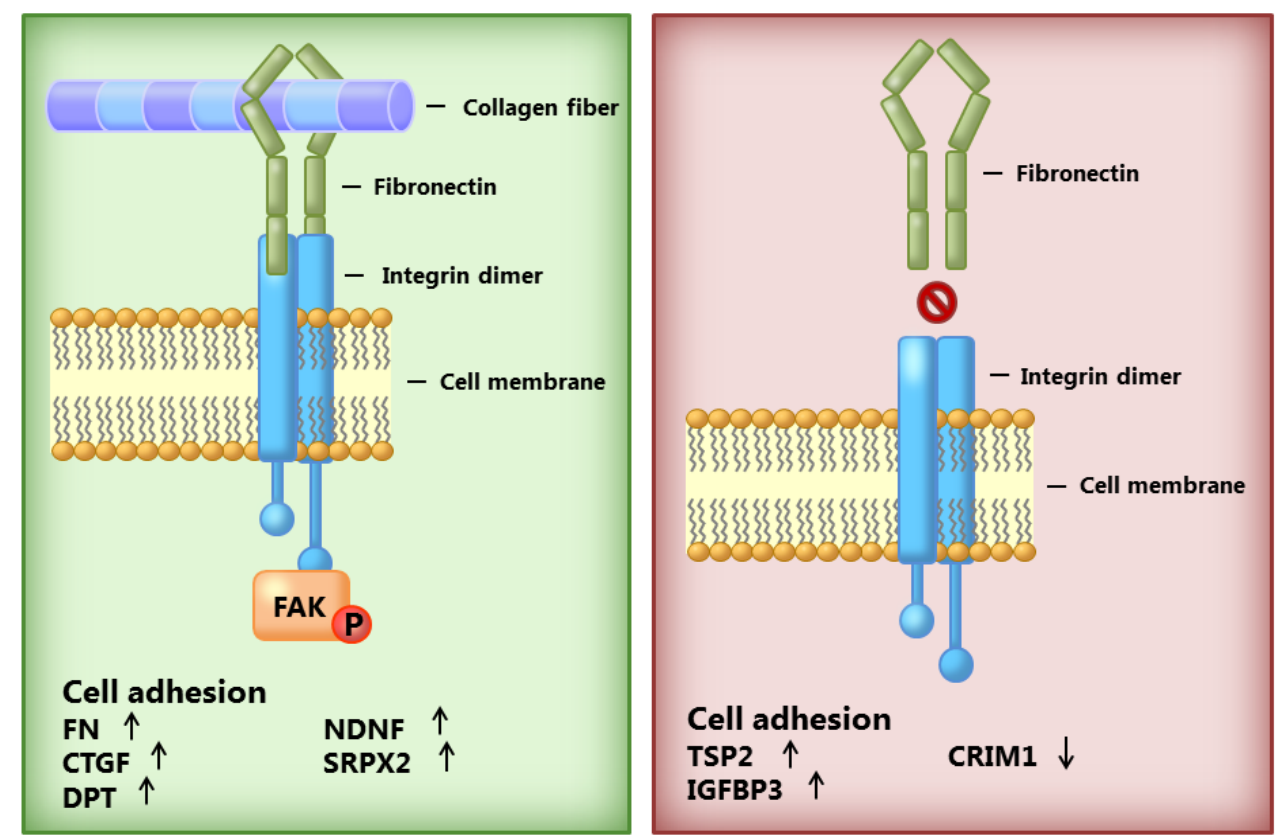

Figure 4. Hypothetical schematic representation of the effects of glycoproteins within the extracellular matrix on cell adhesion in dilated cardiomyopathy. Green framing represents stimulation of the process mentioned, red framing represents inhibition. 